Accepted to the Astrophysical Journal

Preprint typeset using $\mathrm{LAT}_{\mathrm{E}} \mathrm{X}$ style emulateapj v. 2/16/10

\title{
THERMODYNAMIC LIMITS ON MAGNETODYNAMOS IN ROCKY EXOPLANETS
}

\author{
ERic Gaidos and Clinton P. Conrad \\ Department of Geology and Geophysics, University of Hawaii at Manoa, Honolulu, HI 96822 \\ AND \\ Michael Manga ${ }^{1}$ And John Hernlund \\ Department of Earth and Planetary Science, University of California at Berkeley, Berkeley, California, USA 94720. \\ Accepted to the Astrophysical Journal
}

\begin{abstract}
To ascertain whether magnetic dynamos operate in rocky exoplanets more massive or hotter than the Earth, we developed a parametric model of a differentiated rocky planet and its thermal evolution. Our model reproduces the established properties of Earth's interior and magnetic field at the present time. When applied to Venus, assuming that planet lacks plate tectonics and has a dehydrated mantle with an elevated viscosity, the model shows that the dynamo shuts down or never operated. Our model predicts that at a fixed planet mass, dynamo history is sensitive to core size, but not to the initial inventory of long-lived, heat-producing radionuclides. It predicts that rocky planets larger than 2.5 Earth masses will not develop inner cores because the temperature-pressure slope of the iron solidus becomes flatter than that of the core adiabat. Instead, iron "snow" will condense near or at the top of these cores, and the net transfer of latent heat upwards will suppress convection and a dynamo. More massive planets can have anemic dynamos due to core cooling, but only if they have mobile lids (plate tectonics). The lifetime of these dynamos is shorter with increasing planet mass but longer with higher surface temperature. Massive Venus-like planets with stagnant lids and more viscous mantles will lack dynamos altogether. We identify two alternative sources of magnetic fields on rocky planets: eddy currents induced in the hot or molten upper layers of planets on very short period orbits, and dynamos in the ionic conducting layers of "ocean" planets with $\sim 10 \%$ mass in an upper mantle of water (ice).
\end{abstract}

Subject headings: planets and satellites; interiors, magnetic fields, tectonics, physical evolution

\section{INTRODUCTION}

Earth distinguishes itself in the inner Solar System with a magnetic dipole generated by a dynamo in its iron core. Mars had a magnetic field of comparable intensity that collapsed early in the planet's history (Arkani-Hamed 2004) and never re-appeared (Lillis et al. 2008). Mercury has a weak (1\% of Earth) global field (Stanley et al. 2005; Christensen 2006). Venus currently lacks a dynamo, and we have no evidence for or against one in its past (Nimmo 2002). At least one massive satellite in the outer Solar System (Ganymede) appears to have a core dynamo (Schubert et al. 1996).

Planets as small as twice the mass of the Earth have been discovered on short-period orbits around other stars (Mayor et al. 2009). A fraction of these transit the parent star, allowing mean densities to be determined. The smallest transiting exoplanet has a mass of $4.8 \pm 0.8 \mathrm{M}_{\oplus}$ and a mean density of $5.6 \pm 1.3$ $\mathrm{g} \mathrm{cm}^{-3}$, consistent with an Earth-like composition (Léger et al. 2009; Queloz et al. 2009). The second smallest $\left(6.6 \pm 0.9 \quad \mathrm{M}_{\oplus}\right)$ has a mean density only one-third that of Earth, indicating it has a thick volatile envelope (Charbonneau et al. 2009). These two planets may rotate synchronously and have effective emitting temperatures of around $2600 \mathrm{~K}$ and $500 \mathrm{~K}$, respectively (that of Earth is $255 \mathrm{~K}$ ). The Kepler spacecraft mission

\footnotetext{
${ }^{1}$ Center for Integrative Planetary Science, University of California, Berkeley
}

(Koch et al. 2010; Borucki et al. 2010) is expected to find many such "hot" massive rocky planets (Selsis et al. 2007; Gaidos et al. 2007).

Do these planets have magnetodynamos? While there have been many theoretical studies of the dynamos in the Earth and smaller Solar System bodies, as well as in the ice and gas giants (Breuer et al. 2009; Stevenson 2009), rocky planets more massive than the Earth have not been considered, although simple dynamo scaling laws have been applied to gas giants on short-period orbits (Greißmeier et al. 2004). Magnetic fields may protect planetary atmospheres against erosion by stellar winds and coronal mass ejections (Greißmeier et al. 2004; Lundkin et al. 2007; Dehant et al. 2007). Interaction between a giant planet's magnetic field and that of the host star has been inferred from corotational chromospheric activity (Shkolnik et al. 2008; Walker et al. 2008) and may produce detectable radio emission (Lecavelier Des Etangs et al. 2009). Ohmic dissipation of the kinetic energy of winds in partially ionized atmospheres has been proposed as an explanation for the "inflated" radii of some short-period gas giants (Batygin \& Stevenson 2010).

For a planet to have a dynamo, it must contain a fluid layer that is sufficiently electrically conducting for the magnetic Reynolds number $\operatorname{Re}_{m}=V L / \lambda>40$, where $V$ and $L$ are a characteristic fluid velocity and length, respectively, $\lambda=1 /\left(\mu_{0} \sigma\right)$ is the magnetic diffusivity, 
$\mu_{0}=4 \pi \times 10^{-7} \mathrm{~N} \mathrm{~A}^{-2}$, and $\sigma$ is the electrical conductivity. In Earth-like planets the conducting fluid is liquid iron or an alloy thereof $\left(\lambda \sim 2 \mathrm{~m}^{2} \mathrm{sec}^{-1}\right.$ Stevenson $(2009))$. The possibility that the oxidation state of planetary material might prevent iron core formation was explored by Elkins-Tanton \& Seager (2008), but we assume that such cores do form. Metallization of silicates and miscibility with $\mathrm{Fe}$ is not expected until a pressure of $20 \mathrm{TPa}$ (Oganov et al. 2005). In the cores of Earth-size planets $\left(L \sim 3 \times 10^{6} \mathrm{~m}\right)$, convective motions as small as $10^{-4} \mathrm{~m} \mathrm{sec}^{-1}$ are sufficient for $\operatorname{Re}_{m}>40$. The temporal variation of Earth's magnetic field, such as westward drift, imply velocities of $10^{-4}-10^{-3} \mathrm{~m} \mathrm{sec}^{-1}$ at the top of the core.

A dynamo also requires a source of energy to maintain convective motions against internal ohmic dissipation. Core convection can be driven by the removal of heat from the core faster than it can be transported by conduction, by the release of latent heat during phase transitions (i.e., solidification of iron), or by the formation of buoyant fluid via the expulsion of light elements during iron solidification. The last two are related to the first because the latent heat of solidification must be removed. Thus dynamo activity is ultimately related to the transport of heat and the temperature contrast across the core-mantle boundary (CMB). That contrast can be maintained either by efficient cooling of the lower mantle (e.g., by descending slabs of former lithosphere during plate tectonics) or by the heating of the core by radioactive nuclides. The absence of a dynamo in Venus has been ascribed to the lack of plate tectonics, which slows cooling of the mantle, as well as the core (Nimmo 2002).

In dynamo theory, the condition for convection is expressed as a requirement on entropy production, rather than energy production, because the latter is unaffected by dynamo dissipation when mechanical energy is converted to heat. The balance between entropy sinks and sources in the core gives the maximum dissipation $\Phi$ by the dynamo. If $\Phi>0$ then a dynamo is permitted. We calculate $\Phi$ using parameterized models of the interior structure and thermal evolution of a planet consisting of an $\mathrm{Fe}$ core and a silicate mantle. We account for, but do not vary, the presence of light elements in the core. We estimate the magnetic Reynolds number and average surface field using previously established scaling laws. We address whether dynamos operate in rocky planets with different masses, surface temperatures, and modes of mantle convection. Figure 1 is a schematic of our simplified planet. In the equations that follow, we employ the following subscripts: 1 (ambient), p (planet or surface), m (mantle), c (core or core-mantle boundary), i (inner core boundary), and 0 (center).

\section{INSIGHT FROM SCALING LAWS}

The manetostrophic approximation assumes that Lorentz forces are balanced by Coriolis forces in the core (Stevenson 2003). This produces a scaling relationship between magnetic field strength (or dipole moment) and core mass, radius, and rotation rate. Alternatively, ohmic dissipation, and hence the strength of the dynamo, are set by the available power Christensen \& Aubert 2006). Analysis of numerous numerical dynamo simulations shows that, in this regime, the RMS strength of the field in the core, $B_{c}$, can be related to a Rayleigh number based on a mass anomaly flow (mass deficit advected per unit time) (Christensen \& Aubert 2006; Aubert et al. 2009; Christensen 2009), and through that to the convective power in the core (Buffett et al. 1996). In the case where the magnetic Prandtl number is much less than one and essentially all the convective power is lost to ohmic dissipation, that relationship can be expressed in terms of a dimensionless convective power $p$

$$
B_{c}=a_{1} \sqrt{\mu_{0} \bar{\rho}} \Omega\left(R_{o}-R_{i}\right) p^{b_{1}}
$$

where $a_{1}$ and $b_{1}$ are dimensionless parameters, $\bar{\rho}$ is the mean density of the core, $\Omega$ is the angular rotation rate of the planet, $R_{o}$ and $R_{i}$ are the outer and inner boundaries of the convecting zone, and $p=\phi \bar{T} /\left[\Omega^{3}\left(R_{c}-R_{i}\right)^{2}\right]$. $\phi \bar{T}$ is the available convective power per unit mass, where $\phi=\Phi / M_{c}$ is the entropy available per unit mass and time, and $\bar{T}$ is an effective dissipation temperature. Aubert et al. (2009) find $a_{1} \approx 1.65$ and $b_{1}$ to be almost exactly $1 / 3$. The latter eliminates the dependence on $\Omega$ :

$$
B_{c} \approx a_{1} \sqrt{\mu_{0} \bar{\rho}}\left[\phi \bar{T}\left(R_{c}-R_{i}\right)\right]^{1 / 3} .
$$

Likewise, the magnetic Reynolds number can be found by a relationship between the Rossby number and $p$, giving:

$$
\operatorname{Re}_{m}=a_{2} \frac{\Omega\left(R_{c}-R_{i}\right)^{2}}{\lambda} p^{b_{2}},
$$

where again $a_{2}$ and $b_{2}$ are dimensionless. $b_{2}$ is not necessarily $1 / 3$ and this introduces a slight dependence on $\Omega$. For example, Aubert et al. (2009) find $a_{2} \approx 1.3$ and $b_{2} \approx 0.42$. For the purpose of a simple order-ofmagnitude estimate of $\mathrm{Re}_{m}$, we ignore these complications, set $b_{2}=1 / 3$, producing a new formulation:

$$
\operatorname{Re}_{m} \approx a_{2}^{\prime} \frac{R_{c}-R_{i}}{\lambda}\left[\phi \bar{T}\left(R_{c}-R_{i}\right)\right]^{1 / 3}
$$

For plausible terrestrial values $\left(\phi \sim 100 \mathrm{MW} \mathrm{K}^{-1}\right.$, $\bar{T} \sim 5000 \mathrm{~K}$; Labrosse (2007)), $\operatorname{Re}_{m} \sim a_{2}^{\prime} 10^{4}$. So as long $a_{2}^{\prime} \sim 1$ its exact value will not be critical. Combining Equations 2 and 4 for terrestrial values gives $\operatorname{Re}_{m} \sim 16\left(B_{c} / 1 \mu \mathrm{T}\right)$. Thus if the predicted field in the core is at least a few $\mu \mathrm{T}$, i.e. the surface field (attenuated at least by the cubic distance law of a dipole) is $\geq 1 \mu \mathrm{T}$, then $\operatorname{Re}_{m}>40$. This criterion is only very weakly dependent on planet mass.

The strength of the field at the surface of the planet and beyond will be sensitive to the field's topology, especially the fraction in the dipole component, and will depend on $\Omega$. More rapidly rotating planets (lower Rossby number) will have a stronger dipole component (Christensen \& Aubert 2006). In the case of a pure dipole, the average surface field will be

$$
B_{p} \approx B_{c}\left(R_{c} / R_{p}\right)^{3} .
$$

We estimate the $B_{p}$ and $\operatorname{Re}_{m}$ using Equations 2 , 4 and 5. We conservatively take $\bar{T}$ to be the temperature at the top of the core. We assume that the field is dipoledominated like the Earth. 


\section{MODEL}

\subsection{Interior structure}

A planet is modeled as a homogeneous, fully convecting $\mathrm{Mg} / \mathrm{Fe}$-silicate mantle surrounding a liquid/solid $\mathrm{Fe}$ core. The pressure-density profiles are calculated selfconsistently with the (time-varying) size of the inner core. Third-order Birch-Murnagham (BM) equations of state (EOS) are used for each component. The pressure is:

$$
P=\frac{3}{2} K_{1}\left(x^{7}-x^{5}\right)\left[1+\frac{3}{4}\left(4-K_{1}^{\prime}\right)\left(1-x^{2}\right)\right],
$$

where $x=\left(\rho / \rho_{1}\right)^{1 / 3}$ is the isotropic strain. Values of the ambient density $\rho_{1}$, compressibility $K_{1}$ and its pressure derivative $K_{1}^{\prime}$ for the relevant planetary materials are given in Table 1. Electron degeneracy (Thomas-FermiDirac, or TFD) pressure will be important in the metal cores of massive Earth-like planets. We calculated the TFD contribution to the pressure using the formulation of Zapolsky \& Salpeter (1969) and found that the EOS for liquid and $\epsilon$-phase solid iron $(\mathrm{Fe}(\mathrm{l})$ and $\mathrm{Fe}(\epsilon))$ intersect at about 3.3 TPa. Pressures beyond $3 \mathrm{TPa}$ are not relevant to the range of masses considered here. The density in the liquid part of the core is adjusted by a fixed fraction $\delta \rho / \rho$ to account for the presence of light elements (Li \& Fei 2003).

Our interior model reproduces the central pressure (364 GPa), CMB pressure (136 GPa), and core size $R_{c}(3480 \mathrm{~km})$ of the Earth (Dziewonski \& Anderson 1981), but only if the zero-pressure compressibility for $\mathrm{Fe}(\mathrm{l})$ is $40 \%$ larger than the $106 \mathrm{GPa}$ value reported by Anderson \& Ahrens (1994) based on shock experiments. With this adjustment, the predicted radius of the Earth is $25 \mathrm{~km}(0.4 \%)$ less than the actual value, but this is not surprising because we do not include low density crustal rocks or an ocean. Our dynamo predictions are much more sensitive to the properties of the core than those of the entire planet. Predicted planet radius, core radius, pressure at the $\mathrm{CMB}$, and central pressure are plotted versus total mass in Figure 2 Pressures increase as the core solidifies and contracts.

\subsection{Temperature and density profiles}

The adiabatic temperature profile in the outer convecting core is approximately

$$
T(r) \approx T_{c} \exp \left[\left(R_{c}^{2}-r^{2}\right) / D_{0}^{2}\right],
$$

where the thermal length scale evaluated at the planet center is,

$$
D_{0}=\sqrt{\frac{3 c_{p}}{2 \pi \alpha_{0} \rho_{0} G}},
$$

(Labrosse 2003), $c_{p}$ and $\alpha$ are the heat capacity and thermal expansivity of $\mathrm{Fe}$, respectively, and $G$ is the gravitational constant. The temperature profile deviates from Equation 7 to the extent that $D$, and specifically $\alpha$, vary with depth in the outer core. $\alpha$ must therefore be evaluated appropriately when calculating terms that are sensitive to $D$, i.e. the heat and entropy transported by thermal conduction. Hereafter $D$ (without a subscript) is $D_{0}$.

The density in the core follows

$$
\rho(r) \approx \rho_{c} \exp \left[\left(R_{c}^{2}-r^{2}\right) / L^{2}\right],
$$

where the density scale length is

$$
L=\sqrt{\frac{9 K_{1}}{2 \pi G \rho_{1} \rho_{0}}\left(\ln \frac{\rho_{0}}{\rho_{1}}+1\right) .}
$$

For the Earth, $D \sim 6400 \mathrm{~km}$ and $L \sim 7400 \mathrm{~km}$ and these values are only weakly dependent on planet size. We expect the core radius in planets of Earth-like composition to scale as $M_{p}^{0.25}$ (Sotin et al. 2007; Seager et al. 2007). The $n$th order term in either $r / D$ or $r / L$ will be of order $M_{p}^{n / 4} / 2^{n}$. For planets of a few Earth masses, we must retain terms in expansions with $r / D$ or $r / L$ through fifth order.

If a solid inner core is present, the temperature at the inner-outer core boundary is the intercept between the adiabat and the iron melting point $\tau$. Adopting a Lindemann law for $\tau$,

$$
\frac{\partial \log \tau}{\partial \log \rho}=2\left(\gamma-\frac{1}{3}\right)
$$

where $\gamma$ is the Grüneisen parameter. The application of the Lindemann law to the deep interiors of planets is sometimes viewed as speculative (e.g., Wolf \& Jeanloz (1984)). The assumptions upon which it is derived are not strictly valid for polyatomic systems, and the law is known to sometimes fail for minerals with complex interatomic forces and structures. However, at high pressures and for metals it becomes a better approximation, and hence is widely used to interpret shock data (e.g., Anderson \& Ahrens (1996)). Importantly, it appears to provide a good description of experimental data and first principle calculations for Fe (e.g., Hemlev \& Mao (2001); Huser (2007)) and other metals (e.g. Dai et al. (2002)).

The radial dependence of the solidus becomes

$$
\tau(r)=\tau_{0} \exp \left[-2\left(1-\frac{1}{3 \gamma}\right)\left(\frac{r}{D}\right)^{2}\right] .
$$

where we have used $L^{2} / D^{2}=\gamma$ (Labrosse 2003). Thus, the temperature at the top of the core is uniquely set by the ratio of the inner to outer core radius $\Re=R_{i} / R_{c}$ :

$$
T_{c}=\tau_{0} \exp \left[-\left[1+\Re^{2}\left(1-\frac{2}{3 \gamma}\right)\right] \frac{R_{c}^{2}}{D^{2}}\right] .
$$

$T_{c}$ must decrease as the inner core grows. If the entire core is liquid, then $T_{c}$ is the independent variable. For greater accuracy, we use our interior model to directly calculate the density at the ICB, the melting temperature using Equation[11, and $T_{c}$ using Equation 7 ,

The temperature profile of the inner core will depend on whether it, too, is convecting (Buffett 2009). Such convection may be sufficiently torpid so as not to contribute to the dynamo, but as long as overturn is rapid compared to the timescale of inner core growth 
$\left(10^{9}-10^{10} \mathrm{yr}\right)$, the temperature profile will be an adiabat. This assumption maximizes the transport of sensible heat into the outer core, and hence minimizes the rate of inner core growth.

For a fully convecting core, the heat conducted along the the adiabat at the CMB is (neglecting compressibility effects)

$$
Q_{K} \approx \frac{4 \pi R_{c}^{2} k \alpha_{c} g_{c} T_{c}}{c_{p}}
$$

where $k$ is the thermal conductivity and $g$ is the local gravitational acceleration. If $Q_{c}$, the heat flow from the core to the mantle across the CMB, is less than this, the temperature profile will be sub-adiabatic to the degree required to equalize the heat flows. Whether this layer is completely stratified will depend on non-thermal (i.e., light element) buoyancy forces. In the absence of a growing inner core, however, such a layer will be thermally stratified. Geomagnetic variation limits the thickness of any upper stratified layer in the Earth's core to $<100 \mathrm{~km}$ (Gubbins 2007), if one exists at all (Stanley \& Mohammadi 2008).

If the stratified layer is thin and makes a negligible contribution to the sensible heat flow, the heat flow through it is constant and its temperature profile is simple. The location $\Re_{*}$ and temperature $T_{*}$ of the boundary between the convective and conducting regions is found by simultaneously solving for the heat flow and temperature:

$$
\Re_{*}^{3}=\left(\frac{D_{c}}{R_{c}}\right)^{3} \frac{Q_{c}\left(T_{c}\right)}{8 \pi k D_{c} T_{*}}
$$

and

$$
T_{c}=T_{*}-\frac{Q_{c}\left(T_{c}\right)}{4 \pi k R_{c}}\left(\frac{1}{\Re_{*}}-1\right),
$$

and using Equation [7, i.e.

$$
T_{*}=T_{i} \exp \left[\left(\Re^{2}-\Re_{*}^{2}\right)\left(\frac{R_{c}}{D_{c}}\right)^{2}\right],
$$

where the dependence of $Q_{c}$ on $T_{c}$ must be accounted for (3.4), and we evaluate the thermal scale length at the CMB. In the event that stratification does occur, we compute the entropy terms for the convecting part of the core only. This is done by re-scaling the radius and mass of the core in the entropy equations to the size and mass of the convecting zone. $T_{c}$ becomes the temperature $T_{*}$ at the top of the convecting zone, and we assume that since the adiabat is self-similar, such that $d T_{*} / T_{*} \approx d T_{c} / T_{c}$ (effectively ignoring small differences due to changes in the thickness of the convective zone itself).

\subsection{Equation of entropy production}

The condition for maintenance of convective motions for a dynamo can expressed as a balance between sources and sinks of entropy in the core, e.g Labrosse (2007) and Nimmo (2009):

$$
\Phi+E_{K}=E_{R}+E_{S}+E_{G}+E_{L},
$$

where $E_{K}$ is the entropy sink due to conduction along the adiabat, $E_{R}$ the production of entropy by internal heating; $E_{S}$ that due to core cooling (loss of sensible heat); $E_{G}$ the entropy production from buoyancy generation; and $E_{L}$ the entropy production due to latent heat generated by crystallization of the core. The decay of radioisotopes is a possible internal heat source. ${ }^{60} \mathrm{Fe}$ will decay completely $\left(t_{1 / 2}=2.6 \mathrm{Myr}\right.$ ) (Rugel 2009) before planets finish accreting, but ${ }^{40} \mathrm{~K}\left(t_{1 / 2}=1.25 \mathrm{Gyr}\right)$ may be a heat source in Earth's core (Nimmo et al. 2004). Values of the partition coefficient of $\mathrm{K}$ between silicate and $\mathrm{Fe} / \mathrm{Ni}$ alloy melts have been discrepant between different high pressure experiments, possibly for technical reasons (Li \& Fei 2003), but new results limit the abundance of $\mathrm{K}$ to a few tens of ppm, with no evidence for an increase in solubility with pressure (Corgne et al. 2007). In the absence of radiogenic heating,

$$
\Phi=E_{L}+E_{S}+E_{G}-E_{K} .
$$

We calculate $\Phi$, divide by the outer core mass to obtain $\phi$, and use Equations 2, 5] and 4to estimate the magnetic field intensity and magnetic Reynolds number.

Expressions for entropy production (dissipation) have been derived and discussed extensively in the literature, e.g. Lister (2003). Following Labrosse (2007) and Nimmo (2009) we derive expansions in terms of the size of the inner core. The term from the loss of sensible heat comes form the thermodynamic identity $d S=c_{p} d T / T$ under constant pressure and no heats of reaction. Thus

$$
E_{S}=-\int\left(\frac{1}{T_{c}}-\frac{1}{T}\right) \rho c_{p} \frac{\partial T}{\partial t} d V
$$

where the integration is over the volume of the entire core. In the absence of an inner core, and to fifth order in $R_{c} / D$ and $R_{c} / L$,

$E_{S} \approx-\frac{2}{5} c_{p} M_{c}\left(\frac{R_{c}}{D}\right)^{2}\left[1+\frac{2}{7}\left(\frac{R_{c}}{D}\right)^{2}+\frac{6}{35}\left(\frac{R_{c}}{L}\right)^{2}\right] \frac{d T_{c}}{d t}$,

where $M_{c}$ is the total mass of the core. We assume that subsolidus convection continues the adiabatic temperature profile into the inner core (Buffett 2009) and ignore the effect of small changes in the temperature and pressure length scales due to differences in compressibility and density between the liquid and solid $(\epsilon)$ phases. This assumption maximizes the contribution of the inner core to the entropy and energy terms. In the case of a mostly solid core, a detailed treatment of the heat flow from the inner core would be warranted. However, we shall show that in planets much larger than the Earth, inner cores remain relatively small, or do not grow at all.

The entropic term for the latent heat due to core growth is;

$$
E_{L}=4 \pi R_{i}^{2} \rho_{i} \Delta S\left(\frac{T_{i}}{T_{c}}-1\right) \frac{d R_{i}}{d t},
$$

where $\Delta S$ is the specific entropy of fusion. Assuming that the core grows outwards,

$$
\frac{d R_{i}}{d t}=-\frac{D^{2}}{2 R_{i}(\Delta-1)} \frac{1}{T_{c}} \frac{d T_{c}}{d t},
$$


where $\Delta$ is the ratio of the temperature-pressure slopes of the solidus to the adiabat, evaluated at $R_{i}$. Then,

$E_{L}=-\frac{3}{2} \frac{M_{c} \Delta S}{\Delta-1} \frac{\rho_{i}}{\bar{\rho}} \Re\left(1-\Re^{2}\right)\left[1+\left(\frac{R_{c}}{D}\right)^{2} \frac{1-\Re^{2}}{2}\right] \frac{1}{T_{c}} \frac{d T_{c}}{d t}$,

where the ratio of the density at the top of the inner core to the mean density is obtained directly from the interior model (\$3.1).

The entropic term for the release of buoyant fluid (density deficit $\Delta \rho$ ) during the crystallization of the core is (Labrosse 2007);

$$
E_{G} \approx-\frac{3 \pi G M_{c} \Delta \rho D^{2}}{\Delta-1} \frac{\rho_{i}}{\bar{\rho}} \Re \frac{F}{F^{\prime}} T_{c}^{2} \frac{d T_{c}}{d t},
$$

where $\Delta \rho$ is the density change in the fluid due to the presence of light elements, and $F$ and $F^{\prime}$ are the dimensionless functions

$$
\begin{gathered}
F=\frac{1}{5}-\frac{1}{3} \Re^{2}+\frac{2}{15} \Re^{5}+ \\
\left(\frac{R_{c}}{L}\right)^{2}\left[\frac{2\left(1-\Re^{7}\right)}{21}-\frac{1-\Re^{4}}{6}\right]+ \\
\left(\frac{R_{c}}{L}\right)^{4}\left[\frac{1-\Re^{9}}{27}-\frac{1-\Re^{6}}{18}\right], \\
F^{\prime}=1-\Re^{3}-\frac{3}{5}\left(\frac{R_{c}}{L}\right)^{2}\left(1-\Re^{5}\right)-\frac{3}{14}\left(\frac{R_{c}}{L}\right)^{4}\left(1-\Re^{7}\right),
\end{gathered}
$$

c.f Nimmo (2009). To derive this, we ignore work done by expansion of the core (Lister 2003), and assume that the energy is dissipated at temperature $T_{c}$.

The entropy production by the conduction of heat along the adiabat is

$$
E_{K}=\int_{0}^{R_{c}} 4 \pi r^{2} k\left(\frac{1}{T} \frac{\partial T}{\partial r}\right)^{2} d r
$$

(Lister 2003). This is evaluated to be

$$
E_{K}=\frac{12 k M_{c}}{5 \bar{\rho} D_{c}^{2}}\left(\frac{R_{c}}{D_{c}}\right)^{2}\left(1-\Re^{5}\right) .
$$

To more accurately reflect the temperature profile, to which conduction is sensitive, we have evaluated the temperature scale length at the $\mathrm{CMB}$, rather than the core center. The other terms in the entropy equation depend on $D$ to a lesser degree and are evaluated over the entire core, not just the CMB.

\subsection{Core cooling rate}

We solve for the core cooling rate $d T_{c} / d t$ by balancing the heat flow:

$$
Q_{S}+Q_{L}+Q_{G}=Q_{c},
$$

where the terms correspond to sensible heat, latent heat, gravitational energy, and heat transported through the
CMB, respectively. The left-hand terms are all proportional to $d T_{c} / d t$. The sensible heat term is

$$
Q_{S}=\int \rho c_{p} \frac{\partial T}{\partial t} d V .
$$

Assuming the adiabat continues into the inner core, this is

$Q_{S} \approx c_{p} M_{c}\left[1+\frac{2}{5}\left(\frac{R_{c}}{D}\right)^{2}+\frac{4}{35}\left(\frac{R_{c}}{D}\right)^{4}+\frac{12}{175}\left(\frac{R_{c}^{2}}{D L}\right)^{2}\right] \frac{d T_{c}}{d t}$.

The latent heat term is

$$
Q_{L}=\frac{3}{2} \frac{M_{c} \Delta S \Re}{\Delta-1}\left(\frac{D}{R_{c}}\right)^{2} \exp \left[\left(R_{c} / D\right)^{2}\left(1-\Re^{2}\right)\right] \frac{d T_{c}}{d t},
$$

while the gravitational term is,

$$
Q_{G} \approx-\frac{3 \pi G M_{c} \Delta \rho D^{2}}{\Delta-1} \frac{\rho_{i}}{\bar{\rho}} \frac{F}{T_{c}} \frac{d T_{c}}{d t},
$$

We compare $Q_{c}$ to the heat $Q_{K}$ that can be carried by conduction along the core adiabat:

$$
Q_{K}=\frac{8 \pi R_{c}^{3} k T_{c}}{D_{c}^{2}}
$$

If $Q_{c}>Q_{K}$ then the entire core has thermally driven convection. If $Q_{c}<Q_{K}$ then cooling is insufficient to maintain convection in the entire core and a stratified, conducting layer develops (\$3.2). In our calculation of the rate of sensible heat loss, we have neglected the small corrections due to departures of the temperature profile from the purely adiabatic case.

\subsection{Heat transport in the mantle}

We assume that the mantle boundary layer at the CMB develops independently of the flow in the rest of the mantle and its thickness is controlled by a local Rayleigh number Ra, as opposed to the whole-mantle Ra (Nimmo \& Stevenson 2000). Heat is removed from the core according to;

$$
Q_{c}=4 \pi R_{c} k_{m} \mathrm{Nu}_{c}\left(T_{c}-T_{l}\right),
$$

where $T_{l}$ is the temperature of the lower mantle and $k_{m}$ is the thermal conductivity of the mantle. The Nusselt number is

$$
\mathrm{Nu}_{c} \approx\left(\mathrm{Ra}_{c} / \mathrm{Ra}_{*}\right)^{\delta},
$$

where $\delta \approx 0.3$ (Schubert et al. 2001). For a fluid heated from below,

$$
\mathrm{Ra}_{c}=\frac{\rho g \alpha\left(T_{c}-T_{l}\right) d^{3}}{\kappa \eta_{c}}
$$

where the gravitational acceleration $g$ and viscosity $\eta$ are evaluated at the CMB, and $d=R_{p}-R_{c}$. In the case of a partially stratified layer, we substitute $T_{t}$ for $T_{c}$, where $T_{t}$ is the temperature at the top of the stratified, conducting zone.

The heat transport by subsolidus convection in the mantle is;

$$
Q_{m}=\frac{4 \pi R_{p}^{2} \mathrm{Nu}_{m} k\left(T_{m}-T_{p}\right)}{R_{p}-R_{c}},
$$


where $T_{p}$ is the surface temperature. For a uniform internal heat source,

$$
\mathrm{Ra}_{\mathrm{m}}=\frac{\rho g H \alpha d^{5}}{k \kappa \eta_{m}},
$$

where $H$ is the total specific heat generation (per unit mass). $\eta_{m}$ is evaluated at the mantle reference temperature, taken to be halfway along the adiabat. We make the approximation that heat from the CMB is mixed rapidly and uniformly into the mantle (e.g., by plumes) and thus $H=H_{c}+H_{r}$, where $H_{c}=Q_{c} / M_{m}$, where $M_{m}$ is the mass of the mantle, and the radiogenic heat production $H_{r}$ is given by;

$$
H_{r}=\Sigma_{i} C_{i} h_{i} e^{-t / \bar{t}_{i}},
$$

$C_{i}$ is the initial concentration of the $i$ th radioactive isotope, $h_{i}$ is the specific heat production, and $\bar{t}_{i}$ is the mean life. The concentrations adopted for the long-lived radioisotopes ${ }^{40} \mathrm{~K},{ }^{232} \mathrm{Th},{ }^{235} \mathrm{U}$, and ${ }^{238} \mathrm{U}$ are for the case of an "undepleted" terrestrial mantle (Ringwood 1991) given in Table 2 of Kite et al. (2009). The contribution due to mantle cooling is;

$$
Q_{T}=-c_{m} M_{m} \frac{d T_{m}}{d t},
$$

where $c_{m}$ is the is the specific heat capacity of the mantle.

We adopt the viscosity law for a stress exponent $n=1$ material, appropriate for the plate-tectonic regime (Nimmo \& Stevenson 2000):

$$
\eta_{m}=\eta_{*} \exp \left(b \tau_{m} / T\right),
$$

where $b \approx 17$ (Karato et al. 2001), $\tau_{m}$ is the peridotite solidus, and $\eta_{*}$ is a reference viscosity. In Equation 37, we evaluate $\eta$ at the "film" temperature, which is intermediate that of the lower mantle and top of the core (Manga et al. 2001).

Convection in the silicate mantle may involve a mobile lid (i.e., plate tectonics) or a stagnant lid, and in our Solar System we have Earth-mass examples of each (Earth and Venus). Whether one mode or another will be more likely on larger planets will depend on the relative magnitudes of lithosphere stresses (Valencia et al. 2007), the availablity of water to weaken faults (Nimmo \& McKenzie 1998), surface temperatures (Lenardic et al. 2008), and the formation of buoyant, unsubductable crust (Kite et al. 2009). In our model, the mode of mantle convection controls (a) the temperature boundary condition at the mantle side of the CMB; and (b) the Nussult-Rayleigh number relationship in the mantle.

In the case of a mobile lid, we assume that subducted slabs reach the lower mantle, have a temperature equal to the mean mantle temperature $T_{m}$, and directly cool the core. Thus for Equation 35 we set $T_{l}=T_{m}$. We the use the same $\mathrm{Nu}-\mathrm{Ra}$ relation as in Equation 36 . In the stagnant lid case, the lower mantle temperature is set to

$$
T_{l}=\theta \exp \left[\int_{R_{c}}^{R_{p}} \frac{\alpha g}{c_{p}} d r\right],
$$

where the $\theta$ is the potential temperature of the mantle, and the relationship between mean and potential temperatures is taken to be

$$
\theta=T_{m} \exp \left[-\frac{1}{2} \int_{R_{c}}^{R_{p}} \frac{\alpha g}{c_{p}} d r\right] .
$$

We adopt the relationship between Nusselt number, Rayleigh number, and viscosity contrast $N$ across the conducting lid (Frank-Kamenetskii parameter) given by Solomatov \& Moresi (2000);

$$
\mathrm{Nu}=a N^{-(1+\beta)} \mathrm{Ra}^{\beta} .
$$

where $a \sim 1$, and boundary layer stability analysis shows that for a material with stress exponent $n, \beta=n /(n+2)$ (Solomatov 1995). We use $\beta=0.6$.

\section{RESULTS}

\subsection{Parameter values and a nominal Earth-mass case}

Our numerical experiments revealed that the predicted history of the dynamo in a planet with an Earth-like interior is sensitive to: (1) mantle viscosity; (2) the thermal expansivity of iron (related to its compressibility); (3) the thermal conductivity of Fe; (4) initial core temperature; and (5) the heat flow across the CMB. The first is constrained by the requirement to reproduce the size of and pressures in the Earth's core (\$3.1). We parameterize the last by using different values of the critical Rayleigh number $\mathrm{Ra}_{*}$. Althhough $\mathrm{Ra}_{*}$ is of order $10^{3}$ (Nimmo \& Stevenson 2000; Turcotte \& Schubert 2002), we consider a range of values that encapsulate the complex uncertainties of the thermochemical boundary layer at the CMB.

Mantle viscosity: Our viscosity law requires a solidus and a reference viscosity. We fit a Lindemann melting law to the solidus data of Zerr et al. (1998) for the lower mantle, using the Mg-perovskite equation of state to relate $P$ to $\rho$ : We found a best-fit Grüneisen parameter of 1.45. The reference viscosity of a hydrated Earth-like mantle was adjusted to achieve a present potential temperature $\theta$ of $1750 \mathrm{~K}$. This is the present temperature beneath mid-ocean ridge basalts (1623 K) (Herzberg et al. 2010) to which has been added $130 \mathrm{~K}$ associated with the olivine-spinel transition in the deep mantle (latent heat of reaction $=160 \mathrm{~kJ} \mathrm{~kg}^{-1}$ ) (Ito et al. 1971). The derived reference viscosity, $\eta_{*}=6 \times 10^{19} \mathrm{~Pa}$ s, is somewhat above inferred values for the terrestrial asthenosphere where partial melting may occur (Solomatov \& Moresi 2000; Dixon et al. 2004; Fieldskaar \& Cathles 2007; Bills et al. 2007; James et al. 2009). The viscosity of a dehydrated mantle is expected to be significantly higher. The effect of water on viscosity depends on the dominant creep mechanism, and the partitioning of water amongst the mineral phases that are present. Hydration of olivine in the upper mantle could lower the viscosity by a factor of $\sim 500$ (Hirth \& Kohlstedt 1996), but the degree of hydration, and concomitent decrease in viscosity, could be much less in the lower perovskite mantle (Bolfan-Casanova et al. 2000). We selected a value based on the requirement that a simulation of Venus $\left(0.815 \mathrm{M}_{\oplus}\right.$ and $\left.\mathrm{T}_{p}=785 \mathrm{~K}\right)$ predicts no dynamo at the present time. [The slow 
rotation of Venus cannot be solely responsible for the absent dynamo (Zhang \& Zhang 1995).] If Venus has had a stagnant lid over its entire history, then the required viscosity enhancement for the Venusian dynamo to cease by $4.5 \mathrm{Gyr}$ is a factor of 6 . If its lid was primarily mobile, then the required viscosity enhanceent is a factor of 200 . We conservatively adopt a factor of 10 , recognizing that higher values are possible.

Fe thermal conductivity: Thermal conduction competes with advection in removing heat from the core. Higher thermal conductivity will suppress convection, and thus the dynamo, and will stratify some or all of the liquid core. The thermal conductivity of iron is expected to depend on pressure, temperature, and the presence of light elements, but not on temperature (Berman 1976). The value often used in core models is $50-60 \mathrm{~W} \mathrm{~m}^{-1} \mathrm{~K}$ (Labrosse 2003). Thermal conductivity can be determined from measurements of electrical conductivity using the Wiedemann-Franz-Lorenz (WFL) law. Assuming the WFL law applies at high pressure, electrical conductivity measurement at $140 \mathrm{GPa}$ (Keeler \& Mitchell 1969) and $200 \mathrm{GPa}$ (Bi et al. 2002) imply $k \sim 100 \mathrm{~W} \mathrm{~m}^{-1} \mathrm{~K}^{-1}$, c.f., Anderson (1998). In contrast, Stacey \& Loper (2007) argue for a value of 28$29 \mathrm{~W} \mathrm{~m}^{-1} \mathrm{~K}^{-1}$ based on the expectation that scattering of electrons by $3 \mathrm{~d}$ orbitals (which contributes to resistivity) will increase faster with pressure than the densities of $3 \mathrm{~d}$ and $4 \mathrm{~s}$ states (which contribute to conductance). The electrical conductivity measurements of Matassov (1977) show that light elements can significantly reduce thermal conductivity (Anderson 1998), e.g., a factor of two reduction for $\mathrm{Fe}_{3} \mathrm{Si}$ (Manga et al. 1996). A low value of $k$ is an alternative mechanism to radiogenic heat to avoid core stratification (Stacev \& Loper 2007).

Initial core temperature: We presume that the cores of more massive planets will be initially hotter because of the conversion of a greater amount of gravitational potential energy into heat. A hypothetical planet with a small core that grows concurrently by the descent of iron from a cold surface will have a final temperature $T_{0} \approx\left(3 G M_{p}\right)\left(10 R_{p} c_{p}\right) \sim 22100\left(M / M_{\oplus}\right)^{2 / 3} \mathrm{~K}$. These core temperatures are unrealistic because much of the gravitational potential energy will be dissipated in and carried back to the surface by a partially molten silicate mantle. Instead, we set the initial temperature at the top of the core relative to the melting temperature of $\mathrm{Mg}$-perovskite $\left(\mathrm{MgSiO}_{3}\right)$, effectively the liquidus temperature of the mantle, at the CMB. Core temperatures reaching this would result in the formation of a massive magma ocean whose low viscosity would quickly cool a hotter core. Alternatively, our initial state can be considered the epoch when any such magma ocean solidifies. The experimental data and $a b$ initio calculations of the $\mathrm{MgSiO}_{3}$ melting temperature (Stixrude et al. 2009) show that $\tau$ is well described to $136 \mathrm{GPa}$ by the relationship $\tau \approx 5400(\mathrm{P} / 136 \mathrm{Gpa})^{0.367}$. This is equivalent to a Lindeman law and $\gamma=1.44$, c.f. Akaogi \& Ito (1993), and we simply extrapolate this to higher pressures. The mantle is given its steady-state temperature as its initial temperature.
Figure 3 plots the predicted inner core size and the surface magnetic field of the Earth at $4560 \mathrm{Myr}$ for different values of the initial core temperature (from $100 \mathrm{~K}$ above to $500 \mathrm{~K}$ below the $\mathrm{MgSiO}_{3}$ melting temperature), critical Rayleigh numbers between 460 and 3610, and three different values of the thermal conductivity $\left(30,35\right.$, and $\left.40 \mathrm{~W} \mathrm{~m}^{-1} \mathrm{~K}^{-1}\right)$. We calculate a present surface-average terrestrial field strength using a dipole moment of $8 \times 10^{22} \mathrm{~A} \mathrm{~m}^{2}$. To compare with the partially non-dipolar field of the Earth, we multiply our model predictions by $80 \%$ (Lowes 2007). Because $80 \%$ is the measured dipole fraction at the surface, and the fraction at the core will be smaller, our predictions should over-estimate the strength of the Earth's current field. At $k>45 \mathrm{~W} \mathrm{~m}^{-1} \mathrm{~K}^{-1}$ the core becomes partly stratified, inconsistent with observations of magnetic field variability (Gubbins 2007; Stanley \& Mohammadi 2008). At lower values of $k$, the present core size and magnetic field strength are can be reproduced by combinations of a range of critical CMB Rayleigh numbers and initial core temperatures. We adopt $k=35 \mathrm{~m}^{-1} \mathrm{~K}{ }^{-1}, R a_{*}=1100$, and an initial core temperature of $275 \mathrm{~K}$ below the $\mathrm{MgSiO}_{3}$ melting temperature at CMB pressure. We use these parameter values for all subsequent calculations.

Figure 4 shows the calculated 4.56 Gyr evolution of a $1 \mathrm{M}_{\oplus}$ planet. The mantle potential temperature decreases by $200 \mathrm{~K}$, consistent with paleothermometry based on the chemistry of Archean and Proterozoic non-arc basalts (Herzberg et al. 2010). The temperature contrast across the CMB decreases until the onset of inner core formation, then rises slightly, as the core is heated by latent heat from the inner core, to a present value of around $1600 \mathrm{~K}$, only slightly higher than current estimates (Tateno et al. 2009). Calculated mantle heat flow, radiogenic heat flow, and core heat flow are all consistent with current estimates (Buffett 2002; Lav et al. 2008), but the Urey number at $4.56 \mathrm{Gyr}$ is $\sim 0.8$, significantly higher than in some models (Korenaga 2008). The predicted evolution of the surface field (or the equivalent dipole moment) is comparable to that of other models (Labrosse 2007; Aubert et al. 2009; Breuer et al. 2009). It also does not conflict with paleomagnetic data, represented by a filtered sample of the IAGA paleointensity database (Biggin et al. 2009) as well as three recent measurements in $3.45 \mathrm{Ga}$ rocks (Herrero-Bervera \& Mojzsis 2009; Tarduno et al. 2010).

\subsection{Inner cores versus iron snow}

Whether a core grows outward from the center of the planet, or inwards from the top of the core depends on $\Delta$, the ratio of the pressure-temperature slopes of the adiabat and solidus. This value will vary with depth in the core, but is prudently evaluated at the inner core boundary or, when no core is present, at the center of the planet. For an adiabatic temperature profile,

$$
\Delta=\frac{2(3 \gamma-1) K_{1}\left(1+\ln \left(\rho_{i} / \rho_{0}\right)\right)}{\gamma\left[d P / d\left(\rho / \rho_{1}\right)\right]} .
$$

The zero-pressure compressibility $K_{1}$ appears as a linear factor in the EOS, therefore $\Delta$ ultimately depends only 
on the Grüneisen parameter $\gamma$, the pressure-derivative terms in the EOS, and the pressure at the inner core boundary. Because gravity is comparatively low in the core, the pressure is relatively insensitive to the size of the core, and the total mass of the planet is the most important parameter. $\Delta$ will decrease with mass due to the increasing incompressibility of Fe (including the TFD effect) at high pressures. $\Delta<1$ for planets larger than 2.5-3 $\mathrm{M}_{\oplus}$ (Figure 5).

Figure 6] shows the logarithmic ratio of the temperature to the melting point versus depth in the cores of 1-10 $\mathrm{M}_{\oplus}$ planets. An unimportant constant has been ignored in each case. The minima in these curves is where iron will first solidify. Above $2 \mathrm{M}_{\oplus}$ the minimum shifts from the center (corresponding to inner core growth) to a position near or at the CMB. In these cases, iron "snow" will freeze out at or near the top of the core. Except under an exceptionally narrow range of conditions when the temperature coincides with the solidus throughout much of the core (Figure 6), iron crystals or slabs will first form at or near the top of the core and sink, and the resulting release of light elements and transport of latent heat will stabilize the liquid core and shut down convection (Williams 2009). This effect has been discussed for several small bodies in the Solar System (Hauck et al. 2006; Stewart et al. 2007; Chen et al. 2008; Bland et al. 2008).

Because there is no net release of latent heat, the core will continue to cool and iron will crystallize over an expanding depth range (Figure 6). Double diffusive convection can drive a "fingering" instability in a stratified fluid where two gradients, e.g. a thermal gradient and compositional gradient, oppose each other, but are associated with substantially different diffusivities (Huppert \& Turner 1981; Huppert \& Sparks 1984). Double diffusive convection has been included in a recent model of Mercury's dynamo (Manglik et al. 2010). In our case, the release and absorption of latent heat by crystallizing, sinking, and melting grains of iron will create a slightly subadiabatic temperature gradient, and buoyancy forces due to temperature will not play a role. Double diffusive convection will only occur if (a) the diffusion of the light elements is faster than the iron crystals, and (b) the crystals are well-coupled to the fluid, i.e. there is a high density of nucleation sites and crystals are small. Even if convection ensues, it will do so in discrete layers each of which has a Rayleigh number of order the critical value (Huppert \& Sparks 1984). Boundary layer theory for finite-amplitude convection (Turcotte \& Schubert 2002) shows that the magnetic Reynolds number of each layer will be of order $(\kappa / \lambda) \mathrm{Ra}^{2 / 3}$, or $\sim 10^{-3}$, well below that required to sustain a dynamo. If the crystals are not coupled to the fluid, no convective instability will occur. and instead crystals will steadily settle through the fluid. It is not clear that a dynamo occurs under these conditions, either.

\subsection{Variation in the dynamo history of Earths and super-Earths}

For a given total mass, the mass of a planet's core may differ as a result of variation in the ratio of $\mathrm{Fe}$ to $\mathrm{Si}$ in the primordial disk (Neves et al. 2009), the efficiency of core formation (Elkins-Tanton \& Seager 2008) or impact stripping of the mantle (Benz et al. 1988, 2007). The ability of impacts to remove substantial amounts of the mantle from Earth-mass and larger planets might be limited, and thus massive Mercurys may not be plausible (Marcus et al. 2010). Figure 77a shows the predicted dynamo history of Earth-mass planets with core sizes between 0.5 and 1.5 times that of the Earth. The average magnetic field is higher on planets with larger cores, mostly due to the smaller $r^{-3}$ attenuation of the dipole field outside the core.

The initial abundance of heat-producing radioactive nuclides $\left({ }^{40} \mathrm{~K},{ }^{232} \mathrm{Th},{ }^{235} \mathrm{U}\right.$, and $\left.{ }^{238} \mathrm{U}\right)$ may also vary between planets and between systems as a result of chemical and thermal processing of solids in planet-forming disks, and galactic chemical evolution (Kite et al. 2009). $\mathrm{K}$ is a volatile element and the abundance of ${ }^{40} \mathrm{~K}$ will vary with the location of a planet's accretion zone, with those planets (e.g. Mars) forming further out having a higher abundance (Lodders \& Fegley 1997). The Galactic abundances of these isotopes relative to major planetforming elements such as $\mathrm{Si}$ are predicted to have decreased substantially with time, and planets of a given age may accrete with substantially different inventories, but the difference between planets of different ages observed at the present time is much smaller (Kite et al. 2009). Figure 7b shows the predicted dynamo evolution for planets with $50 \%$ and $150 \%$ of the Earth's estimated initial inventory of radionuclides. The predicted surface field is slightly lower with a higher abundance of radionuclides, but is relatively insensitive to this parameter

Figure 8 plots the predicted dynamo history of 1-10 $\mathrm{M}_{\oplus}$ Earth-like planets with and without plate tectonics, and surface temperatures of $288 \mathrm{~K}$ (Earthlike) and $1500 \mathrm{~K}$ (the solidus of basalt). Planets much larger than $1.5 \mathrm{M}_{\oplus}$ with stagnant lids do not produce dynamos and even more massive planets have lower mantle temperatures that exceed the estimated peridotite solidus. Correct modeling of planets with internal magma oceans is beyond the scope of this study and we terminate those runs. Only planets smaller than $2.5 \mathrm{M}_{\oplus}$ form inner cores $\left(<2 \mathrm{M}_{\oplus}\right.$ for those with stagnant lids), and the elapsed time before an inner core appears, corresponding to a rapid increase in the strength of the magnetic field, increases with planet mass. The strength of the magnetic field at any given age decreases with planet mass. On Earth-mass planets with elevated surface temperatures, the strength of the magnetic field is higher and the interval before the onset of core formation is briefer.

Planets more massive than $2 \mathrm{M}_{\oplus}$ (like CoRoT-7b) have long-lived dynamos produced by core cooling, but only if they have a mobile lid. The duration of the dynamo decreases with planet mass and increases with surface temperature. The bottom right-hand panel of Figure 8 extends these trends out to a mass of $10 \mathrm{M}_{\oplus}$.

\section{DISCUSSION AND CONCLUSIONS}

\subsection{Interpretation of trends and implications}

For an inner core to form, enough sensible heat must be removed for the center temperature to reach the solidus. We obtain an approximate timescale $t_{c}$ for this by setting 
$Q_{s}=-Q_{K}$ and performing the appropriate integration. This gives

$$
t_{c} \approx \frac{M_{c} \Delta S}{8 \pi k R_{c}}\left(1-\frac{D^{2}}{R_{c}^{2}} \ln \frac{\tau_{0}(\mathrm{Fe})}{\tau_{c}(\mathrm{pv})}\right) .
$$

where the solidii for $\mathrm{Fe}$ and perovskite are at central and CMB conditions, respectively. For $k=40 \mathrm{~W} \mathrm{~m}^{-1} \mathrm{~K}^{-1}$, and $R_{c} \sim M_{p}^{1 / 4}$ (Figure 2), the first factor is $\approx$ $2 M_{p}^{3 / 4}$ Gyr. The second factor is of order unity: Both solidii increase with planet mass and so we expect $t_{c}$ to increase slightly faster with planet mass than $M_{p}^{3 / 4}$. This is born out by our detailed calculations, i.e a $2 M_{\oplus}$ planet takes more than twice as long to form an inner core than an Earth-mass planet (Figure 8).

A maximum time $t_{s}$ for the complete solidification of the entire core, after the inner core starts growing, is obtained by dividing the total latent heat by the heat flow. (In our cases, the heat flow through the mantle tends to be close to or not much larger than the conduction through the core itself.) In this case, $t_{s}$ is dependent on mass only through the pressure-dependences of certain material properties of the core:

$$
t_{s} \approx \frac{c_{p} \Delta S}{2 k \alpha G} .
$$

For standard values, $t_{s} \sim 50$ Gyr. The iron cores of rocky planets with Earth-like composition will never completely solidify, and solid inner cores never include more than about half of the total core mass in 10 Gyr. Thus, planets that do form inner cores $\left(\leq 2 \mathrm{M}_{\oplus}\right)$ will have dynamos that last longer than the main-sequence life of the parent star.

In planets with masses of 2-3 $\mathrm{M}_{\oplus}$, inner cores do not grow but instead iron condenses as "snow" at the $\mathrm{CMB}$, shutting down convection and the dynamo within 10 Gyr. In yet more massive planets, the core never cools sufficiently for iron to condense. These planets have persistent dynamos produced by core cooling. However, the temperature contrast between the core and the mantle decays, and the heat flow across the CMB eventually falls to the core conduction value. At that point, $\phi=0$ and the dynamo ceases.

At a given planet mass, the surface field $B_{p}$ increases with increasing core size, and a solid inner core formation forms earlier (Figure 7a). The former is a consequence of the cubic distance attenuation law and a shallower $\mathrm{CMB}$, as well as increased heat flow across a thinner mantle. The latter is a result of more rapid cooling, but also higher pressure and a higher solidus at the planet's center. Dynamo history is much less sensitive to the initial abundance of long-lived radionuclides in the planet's mantle (Figure $7 \mathrm{~b}$ ) because of the self-regulating nature of viscosity-dependent mantle convection (Tozer 1972).

$B_{p}$ generally decreases with planet mass. Equations 2 and 5] show why: The intensity of the field in the core $B_{c}$ depends on the available entropy production per unit mass $\phi$ and only weakly on core size. All else being equal, $\phi$ will decrease with mass because the core of a larger planet has less surface area per unit mass and a thicker mantle through which to lose heat. If $\phi \sim R_{c}^{-1}$, this makes $B_{c}$ insensitive to planet mass. The intensity of the field at the surface will decrease as $R_{c}^{-3}$ and thus will roughly scale as $M_{p}^{-3 / 4}$.

Higher surface temperature leads to elevated mantle temperatures and lower viscosities. Although the former lowers the temperature contrast across the CMB, the latter more than compensates because of the exponential decrease of viscosity with temperature. The outcome, increased magnetic field strength and dynamo lifetime, is clearly seen in Figure 8 .

\subsection{CoRoT-7b}

Our model predicts that CoRoT-7b has a dynamo at its estimated age of 1.3-2.2 Gyr (Léger et al. 2009), but only if it has a mobile lid. In this case, the dynamo is driven entirely by core cooling, and no inner core is present. In fact, it is the absence of iron solidification (as "snow") that allows a dynamo to persist. Whether or not plate tectonics operates on CoRoT-7b is debated (Valencia et al. 2007; O'Neill \& Lenardic 2007; Kite et al. 2009), and the presence of a dynamo may be one means of testing this. However, a core dynamo is not the only mechanism that can produce a magnetic field on such an object (see \$5.4).

\subsection{Uncertainties and neglected effects}

High-pressure material properties: The properties of silicates and $\mathrm{Fe}$ at pressures of hundreds or thousands of GPa are very uncertain. Although equations of state become dominated by electron degeneracy pressure at pressures well above $1 \mathrm{TPa}$, other parameters, namely $k$ and $\alpha$, are very important in determining whether a dynamo operates. Aitta (2006) has proposed that iron has a tricritical point at around $800 \mathrm{GPa}$ at which separate solid and liquid phases disappear and the latent heat of fusion vanishes. These conditions are reached at the center of a $2.5 \mathrm{M}_{\oplus}$ planet and at the $\mathrm{CMB}$ of a $6 \mathrm{M}_{\oplus}$ planet (Figure 2).

Mantle structure: We assume a fully convecting mantle: A layered mantle will support a higher temperature contrast for the same heat flow McNamara \& van Keken 2000), and this would tend to suppress dynamo operation. The lowest $150 \mathrm{~km}$ of the Earth's mantle (within the D" layer) may be in the stability field of a post-perovskite (ppv) phase (Oganov \& Ono 2004) and ppv may dominate in sufficiently cool "super-Earth" mantles (Valencia et al. 2009). If a magma ocean existed at the base of the Hadean terrestrial mantle, release of latent heat during crystallization would have suppressed the cooling of Earth's core (Labrosse 2007). Our calculations indicate that the mantles of planets with $\mathrm{M} \geq 2.5 \mathrm{M}_{\oplus}$ and stagnant lids are partially molten. We do not attempt to model the thermal evolution of such objects but they are (at least initially) unlikely to support a dynamo.

Other effects of light element in the core: Our model does not include melting-point depression in the core by light elements, particularly sulfur (S). The effect of these is to delay inner core formation relative to the pure $\mathrm{Fe}$ 
case. In that sense our calculations are optimistic in predicting dynamos. (This is in contrast to the case of small bodies where $\mathrm{S}$ can maintain a dynamo by delaying the freezing of the entire core). However, we choose our initial core temperature based on the present size of the Earth's inner core and thus, relative to Earth, this calibration may cancel some of the effect of S. We include the production of buoyancy flux resulting from the exclusion of light elements from a growing solid core. However we assume that light elements are uniformly mixed into the outer liquid core and do not include possible variations in light element concentrations with planet mass. The liquid silicate-liquid metal partition coefficient of sulfur increases with pressure (Li \& Fei 2003) but no experimental data is yet available for pressures exceeding those at Earth's CMB. If the trend continues to higher mass (and for other elements), the power available for dynamos in the cores of super-Earths may be smaller than our model predicts.

Non-radiogenic sources of mantle heat: Dissipation of tidal strain could be an additional source of heat in the mantles of non-synchronously rotating planets close to their parent stars (Barnes et al. 2010). The timescale for rotational synchronization is a few Myr (Guillot et al. 1996) while that of orbital circularization can be Gyr (Rasio et al. 1996), but both are strongly dependent on semimajor axis. Sufficiently intense heating during this period could lead to a long-lived thermal anomaly in a mantle that influences the transport of heat much like a stagnant lid. We also ignore the potential complication of heating by late giant impacts (Roberts et al. 2009; Arkani-Hamed \& Olson 2010).

Effect of the stellar magnetic field: In the absence of an ionosphere, the ambient magnetic field of the host star $B_{*}$ could reach the planet's core: A sufficiently strong applied field will inhibit convective motion in transverse directions. This will occur when the Stuart number

$$
\mathrm{St}=\frac{\sigma B_{*}^{2} R}{\rho v} \gg 1 .
$$

Using the definition of $\operatorname{Re}_{m}$ we re-write this as:

$$
\frac{\sigma B_{*}^{2} R^{2}}{\rho \lambda} \gg \operatorname{Re}_{\mathrm{m}} .
$$

For typical values $\sigma \sim 5 \times 10^{5} \mathrm{~S} \mathrm{~m}^{-1}, \rho \sim 10^{4} \mathrm{~kg} \mathrm{~m}$, $\lambda \sim 2 \mathrm{~m}^{2} \mathrm{sec}^{-1}, L \sim 3 \times 10^{6} \mathrm{~m}$, the condition becomes $B_{*} \gg 60 \operatorname{Re}_{m}^{1 / 2} \mathrm{nT}$. For $\operatorname{Re}_{m} \sim 10^{3}$ the requirement becomes $B_{*} \gg 2 \mu \mathrm{T}$. $B_{*} \sim 100 \mu \mathrm{T}$ near the surface of the Sun: Assuming that the stellar magnetic field decreases with the square of the distance (because of interplanetary plasma), $S t \gg 1$ only within $0.1 \mathrm{AU}$. Thus inhibition of convection could occur in the cores of the very closest planets, although the increased temperature and conductivity of surface rocks at such proximity to the star may block the stellar field (see \$5.4).

\subsection{Other sources of magnetic fields}

In a planet is embedded in an ambient magnetic field from its star that varies with orbital period $P$, eddy cur- rents will generate an induced field if a layer is sufficiently thick $(d)$ and conducting. The condition for a substantial induced field is (Stevenson 2003)

$$
d \sigma \gg \frac{1}{2 \pi \mu_{0} R_{p}} .
$$

For an Earth-size planet where $d$ is in $\mathrm{km}$ and $P$ in days, the required conductivity $\sigma$ is $\gg 2 P d^{-1}\left(R_{p} / R_{\oplus}\right)^{-1} \mathrm{~S} \mathrm{~m}^{-1}$. The conductivity of anhydrous molten silicates is $0.1-1 \mathrm{~S} \mathrm{~m}^{-1}$ while that of molten carbonates is three orders of magnitude higher (Gaillard et al. 2008). A planet with a magma ocean only a few $\mathrm{km}$ deep on a planet with a very short-period orbit such as CoRoT-7b (0.85 d) could have an induced field.

Uranus and Neptune have magnetic fields that are thought to be generated in a layer of high pressure water (ice) (Cavazzoni et al. 1999). The ionic conductivity of planetary ices is about $2 \times 10^{3} \mathrm{~S} \mathrm{~m}^{-1}$ at pressures above $40 \mathrm{Gpa}$, conditions that are reached at 0.7-0.8 radii in these planets (Stanley \& Bloxham 2006). We added liquid water and high-pressure ice (Ice VII) layers to the interior model described in $\$ 3.1$ to calculate the pressure at the base of an upper water/ice mantle as a function of total planet mass and water mass fraction (Figure 9). A basal pressure of $40 \mathrm{Gpa}$ is reached on planets of a few $M_{\oplus}$ and ice fractions of $\sim 10 \%$. Such an envelope of water could be easily accomodated by the observed radius $\left(2.7 R_{\oplus}\right)$ of GJ 1214b (Charbonneau et al. 2009) but not by the mass and radius of CoRoT-7b (Valencia et al. 2010).

\subsection{Conclusions}

Our model of a planet consisting of a silicate mantle and pure Fe core reproduces the gross interior structure and thermal history of the Earth. Using the magnetic field strength scaling of Aubert et al. (2009), the predicted evolution of the field strength is also consistent with paleomagnetic inferences and the present value. When applied to Venus, with a stagnant lid, an elevated surface temperature, and enhanced mantle viscosity, our model corrctly predicts the absence of a dynamo at present. We are thus confident in extrapolating the model to rocky planets with approximately Earth-like composition but with different mass, surface temperature, and/or mode of convection.

The model predicts that 1-2 $\mathrm{M}_{\oplus}$ planets with plate tectonics will sustain a dynamo for up to 7 Gyr by core cooling before an inner core forms and the dynamo intensifies at a time that depends on surface temperature, mass, and the relative size of the core. Planets in this mass range with stagnant lids will have dynamos only once an inner core begins to grow. Planets with higher surface temperatures can more easily sustain a dynamo because the resulting higher mantle temperatures lead to a lower viscosity and hence more rapid mantle convection and core cooling.

Planets larger than about $2 \mathrm{M}_{\oplus}$ do not develop solid inner cores because the adiabat is steeper than the 
solidus. In such planets, iron freezes first at or near the core-mantle boundary. The latent heat and any light elements released at the top of the core will not be able to drive a long-lived dynamo. In planets between 2-3 $\mathrm{M}_{\oplus}$, this leads to the premature shutdown of any dynamo within 10 Gyr.

In planets with masses $>3 \mathrm{M}_{\oplus}$, iron never begins to freeze in 10 Gyr. If these planets have plate tectonics, our model predicts that they have relatively weak dynamos driven solely by core cooling but which can last several Gyr, depending on mass and surface temperature. The mantles of planets with stagnant lids in this mass range are hot enough to prevent core cooling and dynamo operation, but they may also be partially molten. This last scenario is not addressed by our model. Our model predicts that CoRoT-7b may have a dynamo, but only if the planet has a mobile lid, and the strength of the field at its surface will be less than that at of the present Earth $(\sim 50 \mu \mathrm{T})$. Advances in our knowledge of the properties of planetary materials at very high pressure will improve our ability to make more quantitative predictions about the intensity and longevity of core dynamos in massive rocky exoplanets.

EG is supported by NSF grant AST-0908419 and NASA grant NNX10AI90G, CPC by NSF grant EAR0914712, and MM and JH by NSF EAR-0855737. Emilio Herrero-Bervera and Julien Aubert helped us with the IAGA paleomagnetic database, and we acknowledge the useful comments by an anonymous reviewer.

\section{REFERENCES}

Ahrens, T. J. 2000, Mineral Physics \& Crystallography: A

Handbook of Physical Constants (Washington, DC: AGU)

Aitta, A. 2006, J. Stat. Mech, P12015

Akaogi, M.. \& Ito, E. 1993, Geophys. Res. Lett. 20, 105

Anderson, O. L. 1998, Phys. Earth Planet. Inter., 109, 179

Anderson, O. L., \& Duba, A. 1997, Anderson, O. L., \& Duba, A. 1997, J. Geophys. Res. 102, 22,659

Anderson, W. W., \& Ahrens, T. J. 1996, J. Geophys. Res., 101, 5627

Anderson, W. W., \& Ahrens, T. J., 1994, J. Geophys. Res., 99, 4273

Arkani-Hamed, J. 2010, Geophys. Res. Lett. 37, L02201

Arkani-Hamed, J. 2004, J. Geophys. Res., 109, E03006

Aubert, J., Labrosse, S., \& Poitou, C. 2009, Geophys. J. Int., 179, 1414

Barnes, R., Raymond, S. N., Greenberg, R., Jackson, B., \& Kaib, N. A. 2010, ApJ, 709, L95

Batygin, K., \& Stevenson, D. J. 2010, ApJ, 714, L238

Benz, W., Anic, A., Horner, J., \& Whitby, J. A., 2007, Space Sci. Rev. , 132, 189

Benz, W., Slattery, W. L., \& Cameron, A. G. W. 1988, Icarus, 74, 516

Berman, B. R. 1976, Thermal conduction in solids, (Oxford: Oxford Univ. Press)

Bi, Y., Tan, H., \& Jin, F. 2002, J. Phys. Condens. Matter, 14, 10849

Biggin, A. J., Strik, G. H. M. A., \& Langereis, C. G. 2009, Earth Planets Space, 61, 9

Bills, B. G., Adams, K. D., \& Wesnousky, S. G. 2007, J. Geophys. Res., 112, B06405

Bland, M. T., Showman, A. P., \& Tobie, G. 2008, Icarus 198, 384

Bolfan-Casanova, N., Keppler, H., \& Rubie, D. C. 2000, Earth Planet Sci. Lett., 182, 209

Borucki, W. J., et al. 2010, Science, 327, 977
Breuer, D., Labrosse, S., \& Spohn, T. 2009, Space Sci. Rev., online first

Buffett, B. A. 2009, Geophys. J. Int., 179, 711

Buffett, B. A. 2002, Geophys. Res. Lett., 29, 1566

Buffett, B. A., Huppert, H., Lister, J., \& Woods, A. 1996, J. Geophys. Res., 101, 7989

Cavazzoni, C., Chiarotti, G. L., Scandolo, S., Tosatti, E., Bernasconi, M., \& Parrinello, M. 1999, Science, 283, 44

Corgne, A., Keshav, S., Fei, Y. \& McDonough, W. 2007, Earth Planet. Sci. Lett., 256, 567

Charbonneau, D., et al. 2009, Nature, 462, 891

Chen, B., Li, J., \& Hauck, S. A. 2008, Geophys. Res. Lett., 35, doi:10.1029/2008GL033311

Christensen, U. R. 2009. Space Sci. Rev., online first DOI: $10.1007 / \mathrm{s} 11214-009-9553-2$

Christensen, U. R., \& Aubert, J. 2006, Geophys. J. Int., 166, 97

Christensen, U. R. 2006, Nature, 444, 1056

Dai, C., Tan, H., \& Geng, H. 2002, J. Appl. Phys. 92, 5019

Dehant, V., et al. 2007, Space Sci. Rev., 129, 279

Dixon, J. E., Dixon, T. H., Bell, D. R., \& Malservisi, R. 2004, Earth Planet Sci. Lett. 222, 451

Dziewonski, A. M., \& Anderson, D. L. 1981, Earth Planet Inter., 25,297

Elkins-Tanton, L. T., \& Seager, S. 2008, ApJ, 688, 628

Fjeldskaar, W., \& Cathles, L. 2007, Terra Nova 3, 393

Gaidos, E., Haghighipour, N., Agol, E., Latham, D., Raymond, S. \& Rayner, J. 2007, Science, 318, 210

Gaillard, F., Mohammed, M., Iacono-Marziano, G., Pichavant, M., \& Scaillet, B. 2008, Science, 322, 1363

Goncharov, A. F., Beck, P., Struzhkin, V. V., Haugen, B. D., \& Jacobsen, S. D. 2009, Phys. Earth Planet. Inter. 174, 24

Greißmeier, J.-M., et al. 2004, A\&A, 425, 753

Gubbins, D. 2007, Earth, Planets, Space, 59, 661

Guillot, T., Burrows, A., Hubbard, W. B., Lunine, J. I., \& Saumon, D. 1996, Astrophys. J., 459, L35

Hauck II, S. A., Aurnou, J. M., \& Dombard, A. J. 2006, J. Geophys. Res., 111, E09008

Hemley, R. J., \& Mao, H.-K. 2001, Int. Geol. Rev., 43, 1

Herrero-Bervera, E., \& Mojzsis, S. J. 2009, AGU Fall meeting

Herzberg, C., Condie, K., \& Korenaga, J. 2010, Earth Planet Sci. Lett., 292, 79

Hirth, G., \& Kohstedt, D. 1996, Earth Planet. Sci. Lett., 144, 93

Huppert, H. E., \& Sparks, R. S. J. 1984, Ann. Rev. Earth Planet Sci., 12,11

Huppert, H. E., \& Turner, J. S. 1981, J. Fluid Mech., 106, 299

Huser, G. 2007, Phys. Plasmas, 12, 060701 10.1063/1.1896375

Ito, K., Endo, S., \& Kawai, N. 1971, Earth Planet. Sci., 4, 425

James, T. S., Gowan, E. J., Wada, I., \& Wang, K. 2009, J. Geophys. Res., 114, B04405

Karato, S.-I., Riedel, M. R., \& Yuen, D. A. 2001, Phys. Earth Planet. Inter., 127, 83

Keeler, R. N., \& Mitchell, A. C. 1969, Solid State Comm., 7, 271

Kite, E., Manga, M., \& Gaidos, E. 2009, ApJ, 700, 1732

Koch, D. G., et al. 2010, ApJ, 713, L79

Korenaga, J. 2008, Rev. Geophys., 46, RG2007, doi:10.1029/2007RG000241

Labrosse, S., Herlund, J. W., \& Coltice, N. A. 2007, Nature, 450, 866

Labrosse, S. 2007, in Encyclopedia of Geomagnetism \& Paleomagnetism, ed. D. Gubbins \& E. Herrero-Bervera (Germany: Springer), 127

Labrosse, S. 2003, Phys. Earth Planet. Int., 140, 127

Lay, T., Hernlund, J., \& Buffett, B. A. 2008, Nature Geosci., 1, 25 Lecavelier Des Etangs, A., Sirothia, S. K., \& Gopal-Krishna, Z. P. 2009, A\&A, 500, L51

Léger, A., et al. 2009, A\&A, 506, 287

Lenardic, A., Jellinek, A. M., \& Moresi, L.-N., 2008, Earth Planet Sci. Lett., 271, 34

Li, J., \& Fei, Y 2003, in Treatise on Geochemistry, Vol. 2, ed. H. D. Holland \& K. K. Turekian (Amersterdam: Elsevier), 521

Lillis, R. J., Frey, H. V., \& Manga, M. 2008, Geophys. Res. Lett., 35, L14203

Lister, J. R. 2003, Physics Earth Planet. Int., 140, 145

Lodders, K., \& Fegley, B. 1997, Icarus, 126, 373

Lowes, F. 2007, in Encyclopedia of Geomagnetism \& Paleomagnetism, ed. D. Gubbins \& E. Herrero-Bervera (Germany: Springer), 310 
Lundkin, R., Lammer, H., \& Ribas, I. 2007, Space Science Rev, 129, 245

Manga, M., Weeraratne, D., \& Morris, S. J. S. 2001, Physics Fluids, 13, 802

Manga, M. \& Jeanloz, R. 1996, Geophys. Res. Lett., 23, 3091

Manglik, A., Wicht, J., \& Christensen, U. R. 2010, Earth Planet. Sci. Lett., 289, 619

Marcus, R. A., Sasselov, D., Hernquist, L., \& Stewart, S. T. 2010, ApJ, 712, L73

Matassov, G. 1977, Ph.D. thesis, Univ. California Davis \& Lawrence Livermore Natl. Lab. Report UCRL-52322.

Mayor, M., et al. 2009, A\&A, 507, 487

McNamara, A. K., \& van Keken, P. E. 2000, Geochem., Geophys., Geosyst. 1, 2000GC000045

Neves, V., Santos, N. C., Sousa, S. G., Correia, A. C. M., \& Israelian, G. 2009, A\&A, 497, 563

Nimmo, F. 2009, Geophys. Res. Lett., 36, L10201

Nimmo, F., Price, G. D., Brodholt, J., \& Gubbins, D. 2004, Geophys. J. Int., 156, 363

Nimmo, F. 2002, Geology, 30, 987

Nimmo, F., \& Stevenson, D. J. 2000, J. Geophys. Res., 105, 11969

Nimmo, F., \& McKenzie, D. 1998, Annu Rev. Earth Planet Sci., 26,23

Oganov, A. R., Price, G. D. Price, \& Scandolo, S. 2005, Z. Kristallographie, 220, 531

Oganov, A. R., \& Ono, S. 2004, Nature, 430, 445

O'Neill, C., \& Lenardic, A. 2007, Geophys. Res. Lett., 34, L19204

Queloz, D., et al. 2009, A\&A, 506, 303

Rasio, F. A., Tout, C. A., Lubow, S. H., \& Livio, M. 1996, ApJ, 470, 1187

Ringwood, A. E. 1991, Geochim. Cosmochim. Acta, 55, 2083

Roberts, J. H., Lillis, R. J., \& Manga, M. 2009, J. Geophys. Res., 114, E04009

Rugel, G., et al. 2009, Phys. Rev. Lett., 103, 072502.

Schubert, G., Turcotte, D. L., \& Olsen, P. 2001, Mantle Convection in the Earth and Planets (Cambridge: Cambridge Univ. Press)

Schubert, G., Zhang, K., Kivelson, M. G., \& Anderson, J. D. 1996, Nature, 384, 544

Seager, S., Kuchner, M., Hier-Majumder, C. A., \& Militzer, B. 2007, ApJ, 669, 1279

Selsis, F., et al. 2007, Icarus, 191, 453

Shkolnik, E., Bohlender, D. A., Walker, G. A. H., \& Collier, C. A. 2008, ApJ, 676, 628
Solomatov, V. S., \& Moresi, L.-N. 2000, J. Geophys. Res., 105, 21,795

Solomatov, V. S. 1995, Geophys. Res. Lett. 1995, Phys. Fluids, 7, 266

Sotin, C., Grasset, O, Mocquet, A. 2007, Icarus, 191, 337

Stacey, F. D., \& Loper, D. E. 2007, Phys. Earth Planet. Inter., 161,13

Stanley, S., \& Mohammadi, A. 2008, Phys. Earth Planet. Inter., 168,179

Stanley, S., \& Bloxham, J. 2006, Icarus, 184, 556

Stanley, S., Bloxham, J., Hutchison, W. E., \& Zuber, M. T. 2005 , Earth Planet Sci. Lett., 234, 27

Stevenson, D. 2009, Space Science Rev., online first.

Stevenson, D. J. 2003, Earth Planet Sci. Lett., 208, 1

Stewart, A. J., Schmidt, M. W., van Westrenen, W., \& Liebske, C. 2007 , Science 316,1323

Stixrude, L., de Koker, N., Sun, N., Mookherjee, M., \& Karki, B. B. 2009, Earth Planet. Sci. Lett., 278, 226

Tarduno, J. A., et al. 2010, Science, 327, 1238

Tateno, S., Hirose, K., Sata, N., \& Ohishi, Y. 2009, Earth Planet. Sci. Lett., 277, 130

Tozer, D. C. 1972, Phys. Earth Planet. Inter. 6, 182

Turcotte, D., \& Schubert, G., Geodynamics (2nd ed.; Cambridge: Cambridge Univ. Press)

Valencia, D., Ikoma, M., Guillot, T., \& Nettelmann, N. 2010, A\&A, in press, arXiv:0907.3067

Valencia, D., O'Connell, R. J. O., \& Sasselov, D. D. 2009 Astrophys. Space Sci. 322, 135

Valencia, P. D., O'Connell, R. J., \& Sasselov, D. D. 2007, ApJ, 670, L45

Vocadlo, L., Alfè, D., Gillian, M. J., \& Price, G. D. 2003, Physics Earth Planet. Interiors, 140, 101

Walker, G. A. H., et al. 2008,A\&A482, 691

Wang, Y., Ahuja, R., \& Johansson, B. 2002, J. Phys. Condens. Matter 14, 7321

Williams, Q. 2009, Earth Planet Sci. Lett., 284, 564

Wolf, G. H., \& Jeanloz, R. 1984, J. Geophys. Res., 89, 7821

Zapolsky, H. S., \& Salpeter, E. E. 1969, ApJ, 168, 809

Zerr, A., Diegeler, A., \& Boehler, R. 1998, Science, 281, 243

Zhang, C. Z., \& Zhang, K. 1995, Earth, Moon Planets, 69, 237 


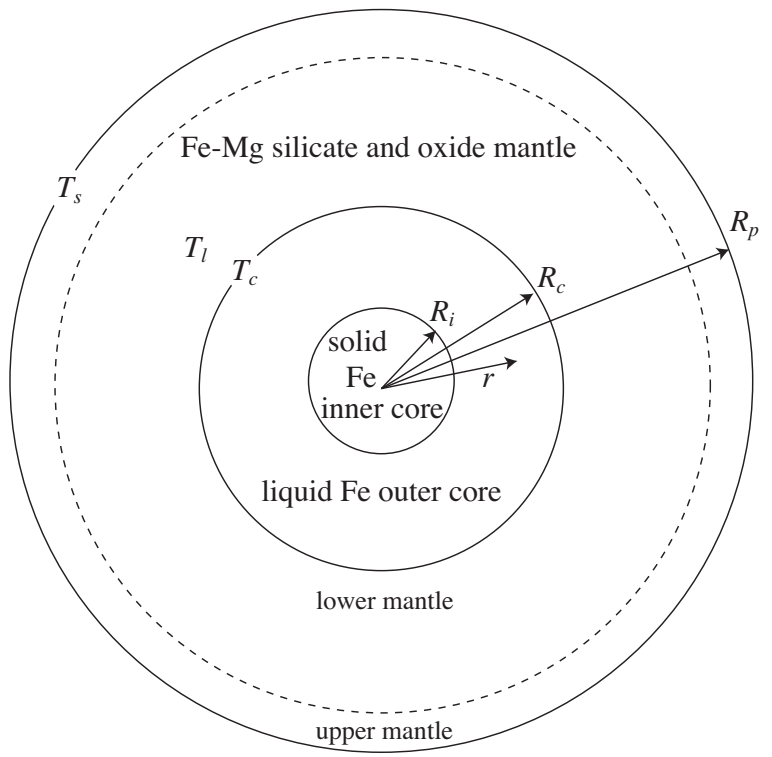

Figure 1. Schematic of our idealized planet.

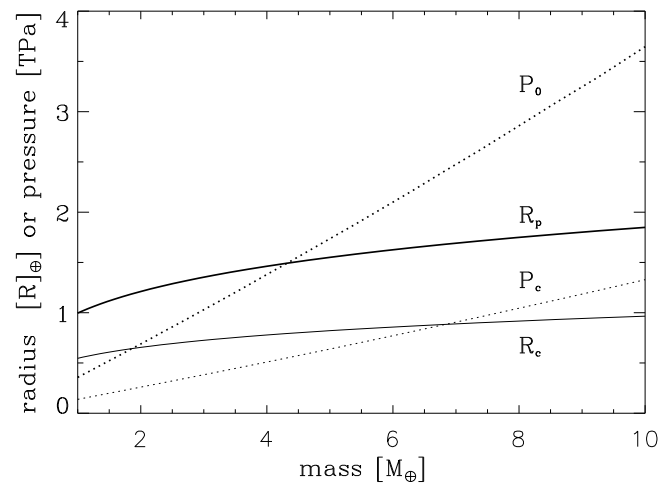

Figure 2. Predicted radius of an Earth-like planet $R_{p}$, and its iron core $R_{c}$, and the central and core-mantle-boundary pressures, $P_{0}$ and $P_{c}$, as a function of total mass. 


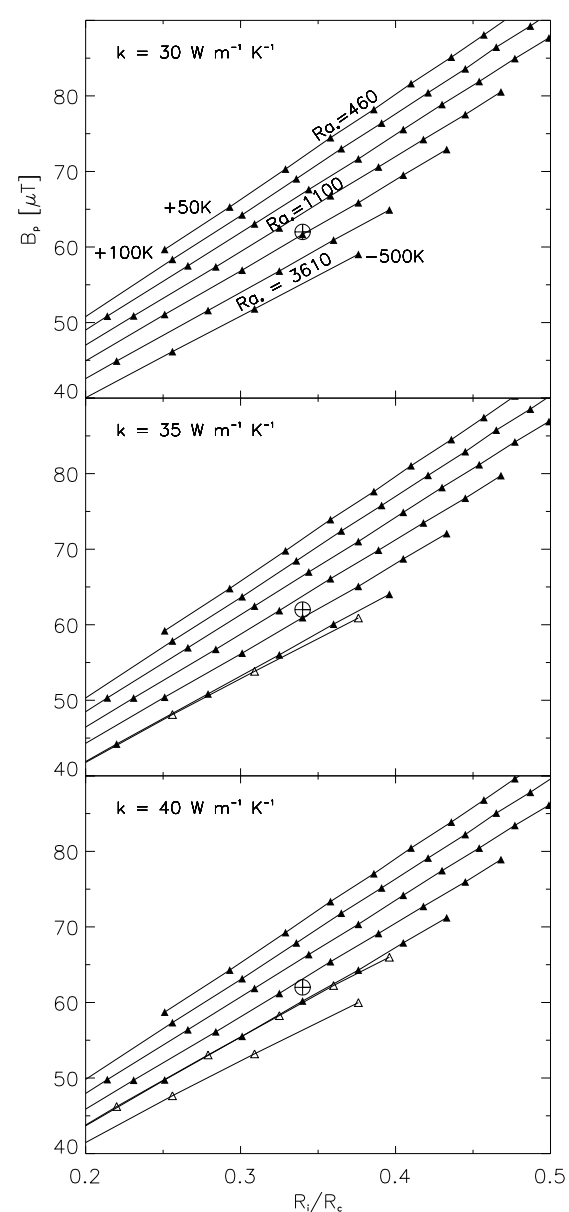

Figure 3. Predicted inner core radius and surface field for 4560 Myr-old Earth-mass planets with different values of initial core temperatures $T_{c}$, critical Rayleigh number $\mathrm{Ra}_{*}$ (which parameterizes the uncertain efficiency of heat transfer across the CMB), and thermal conductivity of Fe $k$. Each curve represents a series of model runs with a given Ra* of (top to bottom) 460, 600, 800, 1100, 1560, 2300, and 3610. Each point along a curve is represents a run with a different value of $T_{c}$. The far right point represents a value $500 \mathrm{~K}$ below the lower mantle liquidus $(5400 \mathrm{~K})$. Each point further to the left represents an increase of $T_{c}$ in $50 \mathrm{~K}$ steps until $100 \mathrm{~K}$ above $5400 \mathrm{~K}$. Solid points represent runs where the entire core is convecting at $4560 \mathrm{Myr}$. Open points represent partially stratified cores. All runs assume plate tectonics and a surface temperature of $288 \mathrm{~K}$. The present inner core radius and mean surface field of the Earth is plotted as the large circle in each pane. The mean was calculated using a dipole moment of $8 \times 10^{22} \mathrm{~A} \mathrm{~m}^{2}$. We assume that the magnetic field is a pure dipole, and thus our model overpredicts the mean field strength at the surface of the Earth. 


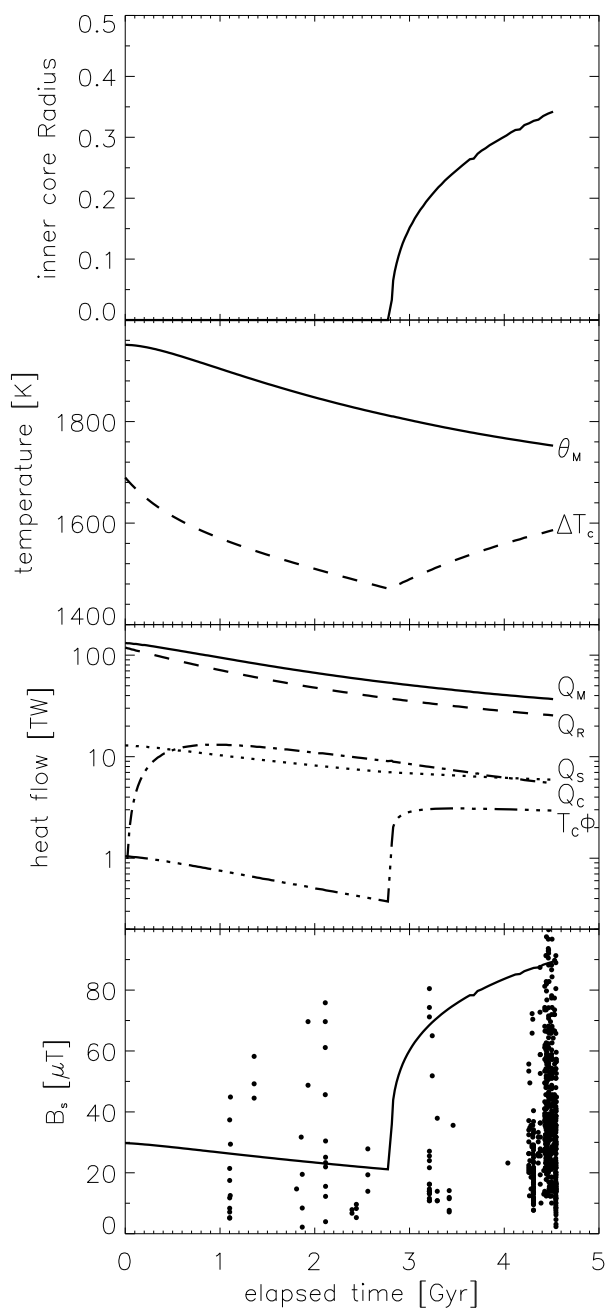

Figure 4. Predicted evolution of the Earth and its dynamo. Top to bottom: inner core radius relative to outer core; mantle potential temperature $\theta$ (solid) and temperature contrast across the CMB $\Delta T_{c}$ (dashed); total surface $Q_{m}$ (solid), radiogenic $Q_{r}$ (dashed), mantle cooling $Q_{s}$ (dot-dash), and core $Q_{c}$ (dotted) heat flows, and $\phi T_{c}$, the power available for the dynamo (dash-triple-dot); average surface magnetic field. In the last pane, 606 measurements from the IAGA paleointensity database (Biggin et al. 2009) and three recent measurements in $3.45 \mathrm{Ga}$ rocks (Herrero-Bervera \& Moizsis 2009; Tarduno et al. 2010) are plotted. Only paleointensity measurements older than $10 \mathrm{Myr}$, having an error less than 50\%, and based on Thellier or Shaw-Thellier techniques with a pRTM check, are shown.

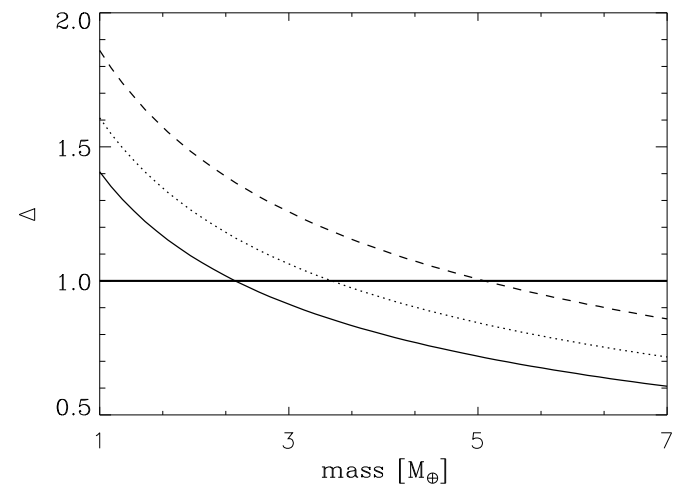

Figure 5. Predicted ratio $\Delta$ of the temperature-pressure slopes of the adiabat versus the solidus in Earth-like planets with different total masses (Earth units). For $\Delta>1$, a solid inner core forms. When $\Delta<1$, iron condenses at the top of the core. In the latter case the release of latent heat stratifies the core and a dynamo is not expected. Solid line: no inner core; dotted line: $50 \%$ inner core by mass; dashed line: nearly completely solid core. 


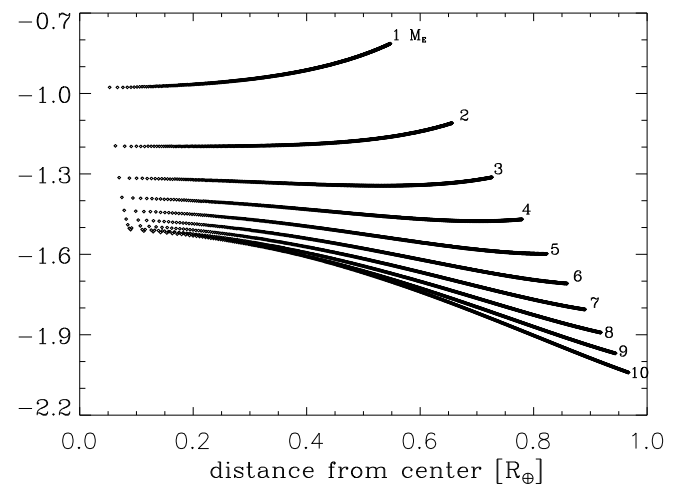

Figure 6. Logarithmic ratio of temperature to solidus with radius in entirely liquid (Fe) cores in planets of 1 (top) to 10 (bottom) Earth masses. Unimportant constants have been ignored. Fe solidification will occur first at the minimum of each curve. Planets with masses less than about $2.5 \mathrm{M}_{\oplus}$ will form solid inner cores. In more massive planets, we predict that iron "snow" will condense near or at the top of the core. The concomitant release of latent heat and the absence of buoyancy forces will stratify the core.

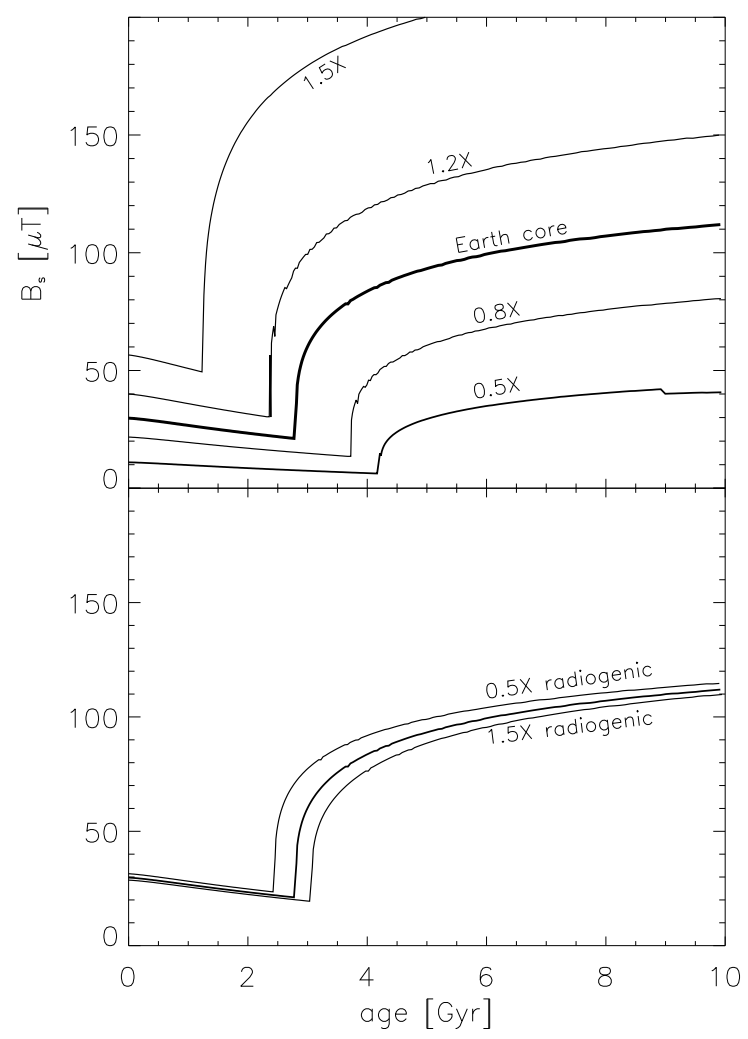

Figure 7. Predicted history of the average surface magnetic field of Earth-mass planets with plate tectonics and surface temperatures of 288 K. Dynamos are assumed to produce pure dipole fields. (a) Top: planets with core masses between 0.5 and $1.5 \times$ that of Earth. (b) Bottom: Planets with an Earth-size core but initial radiogenic element abundances that are 0.5 or $1.5 \times$ the terrestrial case (heavy solid line). 


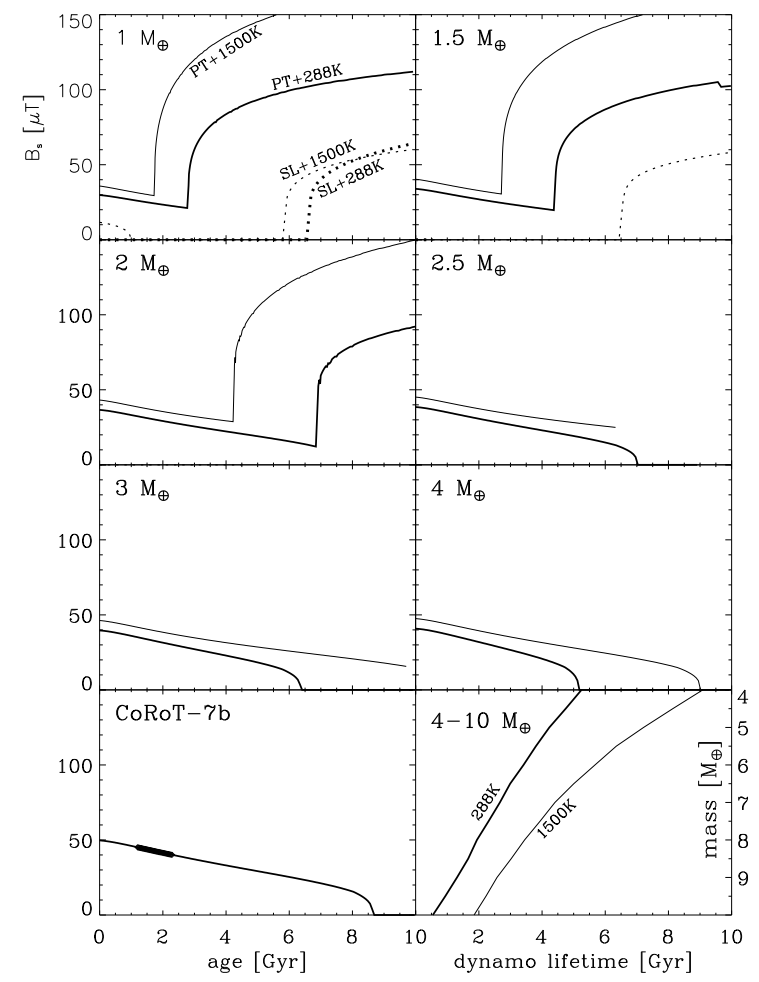

Figure 8. Predicted history of the average surface magnetic field of planets with masses of $1-10 \mathrm{M}_{\oplus}$ and the $\sim 4.8 \mathrm{M}_{\oplus}$ planet CoRoT7b. Absence of a line indicates that the dynamo is inoperative. Dynamos are assumed to produce pure dipole fields. Heavy solid lines: Earth-like planets with plate tectonics and surface temperature of $288 \mathrm{~K}$. Light solid lines: "hot" Earth with plate tectonics and surface temperature of $1500 \mathrm{~K}$. Light dashed lines: Venus-like planets with stagnant lid, 10× elevated mantle viscosity, and surface temperature of $1500 \mathrm{~K}$. Heavy dashed lines: "balmy" Venus with a surface temperature of $288 \mathrm{~K}$. For the case of CoRoT-7b, the surface temperature is set to $1810 \mathrm{~K}$, assuming efficient redistribution of heat (Léger et al. 2009). The thick part of the CoRoT-7 curve spans the range of the system's estimated age (1.2-2.3 Gyr). Lines terminate when $\Delta<1$ and "iron snow" forms at the top of the core, and are absent when no dynamo is present or the simulation was discontinued due to the predicted presence of a magma ocean. The behavior of planets more massive than $4 \mathrm{M}_{\oplus}$ is similar to the $4 \mathrm{M}_{\oplus}$ case. The bottom right-hand panel is the predicted dynamo lifetime as a function of planet mass $>4 \mathrm{M}_{\oplus}$ and the two values of the surface temperature.

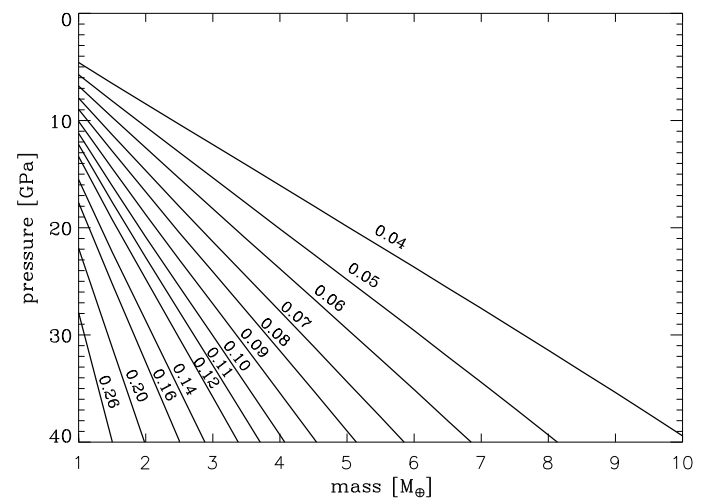

Figure 9. Pressure at the base of a water (ice) mantle in solid planets of varying mass and total water mass fraction. Above 40 GPa, water can be a sufficiently good ionic (proton) conductor $\left(\sim 2 \times 10^{4} \mathrm{~S} \mathrm{~m}^{-1}\right)$ to produce an internal dynamo analogous to those thought to operate in Uranus and Neptune. There is also a minimum temperature requirement that is not considered here. The diameter of GJ 1214b, $\sim 7 M_{\oplus}, 2.7 R_{\oplus}$ (Charbonneau et al. 2009), indicates it has a massive volatile envelope that could easily include the required water mantle. 
Table 1

EOS Parameters

\begin{tabular}{lllll} 
Material & $\rho \mathrm{kg} \mathrm{m}^{-3}$ & $K_{1}[\mathrm{GPa}]$ & $K_{1}^{\prime}$ & Reference \\
\hline Ice/water & 1000 & 2.2 & 4.0 & Sotin et al. (2007) \\
HP ice (VII) & 1460 & 23.9 & 4.2 & Sotin et al. (2007) \\
Mg-Olivine & 3222 & 128 & 4.3 & Sotin et al. (2007) \\
Perovskite & 4260 & 266 & 3.9 & Ahrens (2000) \\
$\mathrm{Fe}(\mathrm{l})$ & 7019 & $154^{\mathrm{a}}$ & 4.66 & Anderson \& Ahrens (1994) \\
$\mathrm{Fe}(\epsilon)$ & 8315 & 165 & 4.97 & Vocadlo et al. (2003) \\
\hline
\end{tabular}

${ }^{a}$ Adjusted $40 \%$ upwards from the Anderson \& Ahrens (1994) value.

Table 2

Thermodynamic Parameters

\begin{tabular}{|c|c|c|c|c|}
\hline Symbol & Parameter [units] & Material & Value & Reference \\
\hline \multirow[t]{2}{*}{$c_{p}$} & specific heat capacity $\left[\mathrm{J} \mathrm{kg}^{-1} \mathrm{~K}^{-1}\right]$ & $\mathrm{Fe}$ & 850 & Wang et al. (2002) \\
\hline & & $\mathrm{MgSiO}_{3}-\mathrm{pv}$ & 1250 & Akaogi \& Ito (1993) \\
\hline \multirow[t]{2}{*}{$\mathrm{k}$} & thermal conductivity $\left[\mathrm{W} \mathrm{m}{ }^{-1} \mathrm{~K}^{-1}\right]$ & $\mathrm{Fe}$ & $30-100$ & Stacey \& Loper (2007) \\
\hline & & $\mathrm{MgSiO}_{3}-\mathrm{pv}$ & $\sim 6$ & Goncharov et al. (2009) \\
\hline$\alpha$ & thermal expansivity $\left[\mathrm{K}^{-1}\right]$ & $\mathrm{MgSiO}_{3}-\mathrm{pv}$ & $3 \times 10^{-5}$ & Anderson \& Duba (1997) \\
\hline$\gamma$ & $\begin{array}{l}\text { Grüneisen parameter } \\
\text { "» }\end{array}$ & $\begin{array}{c}\mathrm{Fe} \\
\mathrm{MgSiO}_{3}-\mathrm{pv}\end{array}$ & $\begin{array}{l}1.5 \\
1.45\end{array}$ & $\begin{array}{l}\text { Labrosse (2007) } \\
\text { Akaogi \& Ito (1993) }\end{array}$ \\
\hline$\Delta \rho / \rho$ & light element density deficit [\%] & $\mathrm{Fe}$ & 10 & Li \& Fei (2003) \\
\hline \multirow[t]{2}{*}{$\Delta S$} & entropy of fusion $\left[\mathrm{J} \mathrm{kg}^{-1} \mathrm{~K}^{-1}\right]$ & $\mathrm{Fe}$ & 118 & Anderson \& Duba (1997) \\
\hline & $" "$ & $\mathrm{MgSiO}_{3}-\mathrm{pv}$ & 130 & Ito et al. (1971) \\
\hline$\lambda$ & magnetic diffusivity $\left[\mathrm{m}^{2} \mathrm{sec}^{-1}\right]$ & $\mathrm{Fe}$ & 2 & Stevenson (2003) \\
\hline$\kappa$ & thermal diffusivity $\left[\mathrm{m}^{2} \mathrm{sec}^{-1}\right]$ & $\begin{array}{l}\mathrm{MgSiO}_{3}-\mathrm{pv} \\
\mathrm{Fe}\end{array}$ & $\begin{array}{l}1 \times 10^{-6} \\
\sim 10^{-5}\end{array}$ & $\begin{array}{l}\text { Turcotte \& Schubert (2002) } \\
\text { calculated }\end{array}$ \\
\hline$\sigma$ & electrical conductivity $\left[\mathrm{S} \mathrm{m}^{-1}\right]$ & $\mathrm{Fe}$ & $5 \times 10^{5}$ & Bi et al. (2002) \\
\hline$\eta_{*}$ & reference viscosity $\left[\mathrm{kg} \mathrm{m}^{-1} \mathrm{sec}^{-1}\right]$ & $\mathrm{MgSiO}_{3}-\mathrm{pv}$ & $6 \times 10^{19}$ & tuning \\
\hline \multirow[t]{2}{*}{$\tau_{0}$} & 1-bar melting point $[\mathrm{K}]$ & $\mathrm{Fe}$ & 1811 & Anderson \& Duba (1997) \\
\hline & liquidus at Earth CMB [K] & $\mathrm{MgSiO}_{3}-\mathrm{pv}$ & 5400 & Stixrude et al. (2009) \\
\hline
\end{tabular}

